Document downloaded from:

http://hdl.handle.net/10251/168794

This paper must be cited as:

Valencia-Sullca, JF.; Boria Esbert, VE.; Guglielmi, M.; Cogollos, S. (2020). Compact Wideband Hybrid Filters in Rectangular Waveguide With Enhanced Out-of-Band Response. IEEE Transactions on Microwave Theory and Techniques. 68(1):87-101. https://doi.org/10.1109/TMTT.2019.2947911

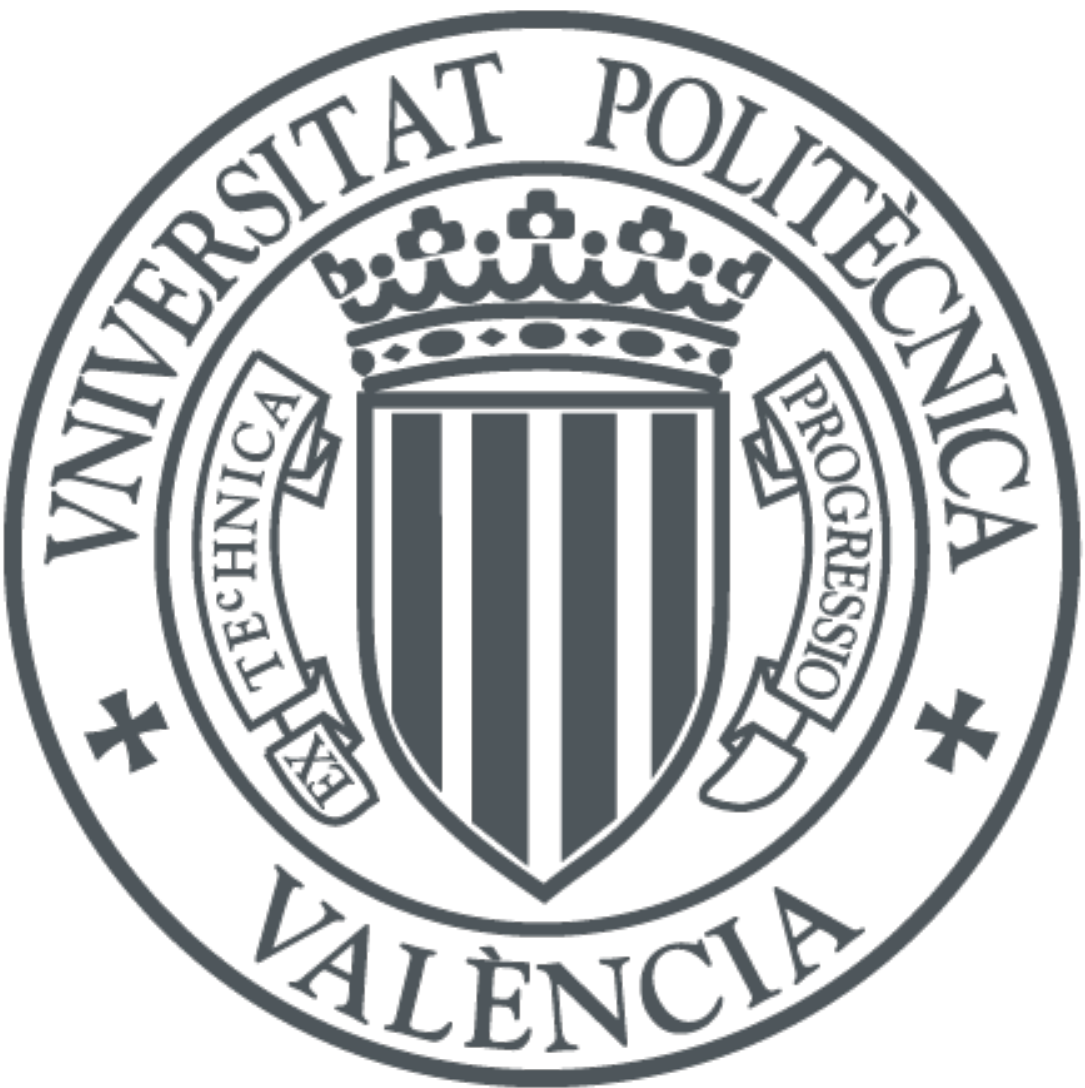

The final publication is available at

https://doi.org/10.1109/TMTT.2019.2947911

Copyright Institute of Electrical and Electronics Engineers

Additional Information 


\title{
Compact Wideband Hybrid Filters in Rectangular Waveguide with Enhanced Out-of-Band Response
}

\author{
Joaquin Valencia, Vicente E. Boria, Fellow, IEEE, Marco Guglielmi, Fellow, IEEE and \\ Santiago Cogollos, Member, IEEE
}

\begin{abstract}
This paper describes the design procedure of a compact wideband waveguide filter, based on stepped impedance resonators $(S I R)$ and a combined usage of capacitive and inductive coupling irises, that provides a significant improvement in the out-of-band response of the filter with respect to the state-ofthe-art. In addition to theory, several filter prototypes have been designed and manufactured. A detailed sensitivity analysis has been also carried out. Finally, simulations and measurements are compared, showing very good agreement, thereby fully validating the new filter topology.
\end{abstract}

Index Terms-Aggressive space mapping, band-pass filters, hybrid (inductive and capacitive) irises, stepped impedance resonator and wideband.

\section{INTRODUCTION}

I $\mathrm{N}$ recent years, the rapid growth in demand for telecommunication services has motivated very significant research in the area of microwave components to reduce size, increase bandwidth, improve out-of-band rejection, and enhance selectivity. In this context, one of the most important components are microwave filters ([1], [2]). The study, analysis and design of microwave filtering structures is indeed one of the oldest areas of research in the microwave field. Wide band filters, in particular, have been traditionally designed in rectangular waveguide as low-pass filters ([3], [4]). Several methodologies and sets of formulas have been developed since the 1970s for the design of both wide and narrow band filters based, for instance, on lumped-element networks [5]-[9].

Based on the early theories developed, microwave filters have been implemented in many different technologies such as metallic waveguide, coaxial and planar technology, just to mention a few ([10], [11]). In this context, a key performance parameter for all microwave filters is the out-ofband performance, especially for frequencies higher than the pass band. The key issue is that if the filters are implemented with coupled resonator structures, or with cascaded lengths of uniform transmission lines, in addition to the desired pass band region, a number of spurious responses appear at (or near to)

Manuscript received February 28, 2019; revised April 26, 2019; accepted August 08, 2019.

This work has been supported by MINECO (Spanish Government) under R\&D project TEC2016-75934-C4-1-R and by Generalitat Valenciana under Santiago Grisolia grant GRISOLIA/2017/073.

J. Valencia, V.E. Boria, M. Guglielmi and S. Cogollos are with Departamento de Comunicaciones, iTEAM, Universitat Politècnica de València, E-46022, Spain (e-mails: joavasu2@teleco.upv.es, vboria@dcom.upv.es, marco.guglielmi@iteam.upv.es, sancobo@dcom.upv.es). multiples of the filter center frequency. Many approaches have indeed been proposed in the past to improve this key aspect of the filter performance. For instance, the use of mixed lumped and distributed components to create hybrid filtering structures has been known since the 1990s [12]. The basic idea is that if the filter is composed of different types of resonators, for instance, the out-of-band performances of the resonators do not contribute constructively to the out-of-band response of the filter, so that an improved performance is obtained.

Many different hybrid filter implementations have indeed been explored. In [13], [14], for instance, a new configuration that uses the $\mathrm{TE}_{01}$ and $\mathrm{HE}_{11}$ modes has been proposed with the objective of improving the out-of-band performance of a dielectric resonator filter. Another approach has been proposed in [15], based on mixed capacitive and inductive couplings, in order to generate transmission zeros near to the pass band of a combline filter to improve the selectivity. Also, in [16] a folded rectangular waveguide filter have been designed with mixed capacitive and inductive irises in order to obtain transmission zeros in the stop band. These last two references use a similar approach to obtain transmission zeros in narrowband designs, namely inductive and capacitive irises to obtain positive and negative couplings. Furthermore, mixed resonator structures have also been discussed in [17], where combline resonators and the $\mathrm{HE}_{11}$ mode dielectric resonator have been combined to improve the out-of-band performance. Another example is [18], where the use of mixed electric and magnetic coupling between the resonators is shown to give an improved selectivity. Also, a hybrid structure using a substrate integrated waveguide (SIW) and coplanar waveguide (CPW) was proposed in [19], with of the objective of improving both the in-band and the out-of-band responses.

More recently, several papers have focused on mixed-mode and hybrid structures in order to improve the pass band and the out-of-band performance. In [20] and [21], a pseudo-elliptic filter has been proposed using mixed-mode (cavity and dielectric) resonators. Also, another hybrid structure with greater selectivity and improvement of the out-of-band response has been discussed in [22], combining coaxial resonators and an evanescent mode waveguide loaded with posts.

Bandpass structures have also been designed using both quarter-mode SIW with mixed coupling [23] and with CPW [24], in order to increase the selectivity and obtain an improved out-of-band response. Yet another approach can be found in [25] and [26], based on the use of lumped elements, 
in order to obtain significant improvements in both the pass band and the out-of-band responses.

Many additional examples of hybrid structures can also be found in the technical literature for wide band filter implementations in planar technology. In [27], for instance, a highly selective wide band bandpass filter is presented, which combines microstrip and CPW sections. On the other hand, a significantly smaller number of contributions can be found in rectangular waveguide technology. In [28], a filter design with a wide pass band covering the $40-60 \mathrm{GHz}$ range is discussed. The solution proposed has a good out-of-band performance, and a wide rejection band. However, the resulting structure, based on the cascaded connection of a bandpass filter and a low-pass section, is rather long.

In the waveguide framework, it is important to recall that, traditionally, band-pass filters have been implemented with inductive irises [29]. Inductive filters, however, present limitations in the design with respect to bandwidth, and unwanted spurious responses. An alternative is the use of capacitive irises as discussed in [30] and [31]. Several advanced contributions for band-pass filters, using capacitive low-pass structures as irises to provide a wide spurious free out-of-band response, can indeed be found in [32] and [33], for instance. The use of capacitive irises in band-pass filters, however, may result in very small gaps leading to high-power limitations.

Few contributions can also be found discussing the design and implementation of waveguide structures using hybrid irises in rectangular waveguide. For instance, in [34] a bandpass filter in $\mathrm{Ku}$ band has been designed using smallaperture irises for coupling the adjacent cavities. Inductive and capacitive irises are used for direct implementation of a C-band filter resonator in [35]. In [36], the use of twosided double-slot capacitive irises (resonant irises with more complex geometry) has been proposed. However, all these filters have been designed only for narrow band applications.

Recently, further solutions have been proposed to improve the out-of-band response of waveguide bandpass filters. In [37], for instance, an inductive filter with dielectric resonators with a good spurious-free band has been proposed. The well-known concept of changing the resonator widths to improve the out-of-band performance has also been exploited in [38] and [39]. In another contribution [40], a structure was proposed to improve the stop-band performance by varying the width of each resonator, as well as the offsets between adjacent resonant cavities. Also, in [41] a structure with capacitive-loaded resonators that can avoid spurious responses was proposed. Furthermore, in [42], an inductive waveguide band-pass filter is presented, which combines inductive irises of different thicknesses, and resonators of different widths, to reduce the bandwidth of the first harmonic pass band, and further improve the out-of-band response.

Moreover, integrated ceramic waveguide filters, with nonuniform width resonators based on a monolithic ceramic structures, have been proposed in [43] and [44], respectively, in order to have a wide spurious-free bandwidth. Additionally, a recent contribution can be found in [45] discussing the design of a filter based on a waveguide loaded with dielectrics of different width, in order to obtain a wide spurious-free frequency range. However, these contributions, and the ones in the previous paragraph, only discuss narrow band structures.

Furthermore, two more contributions can be found in the technical literature that discuss how to improve the stop-band performance of a microwave filter using stepped impedance resonators (SIRs). In [46], SIRs in an empty waveguide coupled through symmetric inductive irises are used, whereas SIRs implemented with integrated ceramic waveguides coupled through inductive posts are used in [47]. Both approaches provide wide spurious-free ranges but only for narrow band bandpass responses. An additional contribution has recently shown how the performance of SIR filters (with a wide band response) can be further improved by using, at the same time, capacitive and inductive coupling irises (hybrid coupled filters) [48]. However, the results presented in [48] were preliminary experimental data of a low-cost and large-size prototype.

The objective of this paper is to contribute to the state-ofthe-art of wide band SIR filters by discussing in detail a filter structure that uses simultaneously capacitive and inductive coupling irises to enhance the out-of-band response. Several prototypes have been designed, manufactured and measured, based on different configurations that provide advantages either to ease the fabrication process, or to achieve simultaneously a reduced footprint and lower insertion losses.

The manuscript is organized as follows. In section II, the target electrical specifications of a wide band filter are given together with the design procedure used for all the prototypes discussed in the paper. In section III, filters with only inductive irises, with only capacitive irises and combining both types are studied, to identify the best location for each coupling window in the hybrid solution. In sections IV and V we studied how to improve the out-of-band response changing the width of the resonators and using SIRs, respectively. Section VI shows a novel wide band filter based on SIRs and combined couplings, which is modified in section VII to produce a simpler configuration with a body and a cover. In section VIII, a novel folded layout leading to a more compact design is successfully validated with experimental results. Section IX includes a tolerance analysis of the three filters manufactured. The main conclusions are finally outlined in section $\mathrm{X}$.

\section{FILTER SPECIFICATIONS}

The design of a microwave filter in rectangular waveguide technology usually starts with a distributed circuit model of the filter (involving lengths of transmission lines and impedance inverters, for instance) that can fulfill the electrical specifications. In Table I we show the electrical specifications that have been used for all filter designs discussed in this paper. The objective of our contribution is to discuss the design procedure of a filter structure that can satisfy the above specifications and provide, at the same time, an out-of-band response that is free from spurious responses in a frequency range, both below and above the pass band, that is as large as possible.

The design process follows at this point the classical approach:

- According to the specifications, the Return Loss $(R L)$ is set and $N$ (order of the filter) is chosen. The normalized 
TABLE I

ELECTRICAL SPECIFICATIONS FOR ALL THE FILTERS DESIGNED.

\begin{tabular}{|l|l|}
\hline \multicolumn{1}{|c|}{ Parameter } & Requirement \\
\hline \hline Bandwidth & $1410 \mathrm{MHz}$ \\
\hline In-band return loss & $>20 \mathrm{~dB}$ \\
\hline Center frequency & $11 \mathrm{GHz}$ \\
\hline $\begin{array}{l}\text { Guide-wavelength } \\
\text { fractional bandwidth }\end{array}$ & $20 \%$ \\
\hline Waveguide WR-90 & $\begin{array}{l}a=22.86 \mathrm{~mm} \\
b=10.16 \mathrm{~mm}\end{array}$ \\
\hline
\end{tabular}

lumped low pass network ( $g_{i}$ values) of the Chebyshev filter is then obtained.

- The distributed model is obtained cascading inverters and transmission lines working with the fundamental $T E_{10}$ mode. The impedance inverters $K_{i, i+1}, i=0, \ldots, N$ have the well-known expressions

$$
\begin{aligned}
\bar{K}_{i, i+1} & =\frac{1}{\sqrt{g_{i} g_{i+1}}} \sqrt{\frac{\pi}{2} \mathcal{W}_{\lambda}}, \quad i=0, N \\
\bar{K}_{i, i+1} & =\frac{1}{\sqrt{g_{i} g_{i+1}}} \frac{\pi}{2} \mathcal{W}_{\lambda}, \quad i=1, \ldots, N-1
\end{aligned}
$$

with $\mathcal{W}_{\lambda}$ being the guided-wavelength bandwidth as defined in [29], and the normalization is performed with respect to the modal characteristic impedance of the $T E_{10}$ mode.

- With inverter values $\left(\bar{K}_{i, i+1}\right.$ or $\left.\bar{J}_{i, i+1}\right)$ the physical dimensions of irises are designed, following any of the available procedures (see [29], [49] and [50]). The electromagnetic (EM) model with real coupling windows and resonators is obtained using the built-in multimode network representation of FEST3D. The wideband behavior of the capacitive or inductive windows is taken into account in this model, and the out-of-band response is therefore included in the design process.

- A final optimization step is carried out to fully meet the filter specifications. This is required in order to recover the in-band response since (1) are approximate formulas.

\section{HYBRID FILTERS}

As already mentioned, inductive irises have been extensively used in the past for the design of (inductive) microwave filters [49]. The classic inductive filter is based on half wavelength resonators that are coupled by inductive irises (see Fig. 1, left). This structure can be used for (relatively) narrowband implementations following the design procedure described in the previous paragraph. However, when the bandwidth increases, the out-of-band response of the filter can be severely degraded.

As it is well known, the appearance of spurious responses at higher frequencies in inductive filters is, in fact, due to the combined effects of the higher order resonances of the resonators, and of the inductive coupling irises that provide more coupling strength as the frequency is increased.

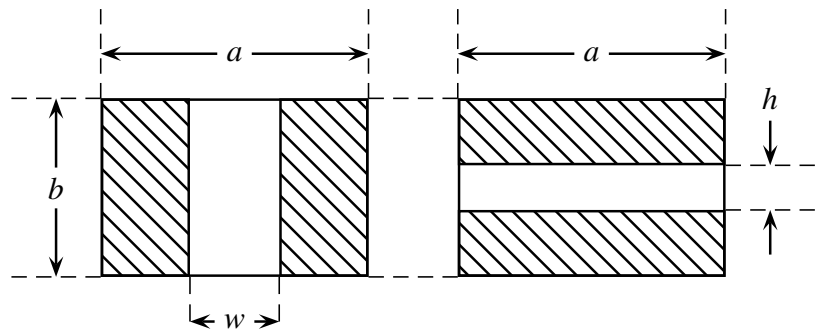

Fig. 1. Typical irises in a rectangular waveguide (front view). Left: centered inductive iris of width $w$. Right: centered capacitive iris of height $h$.

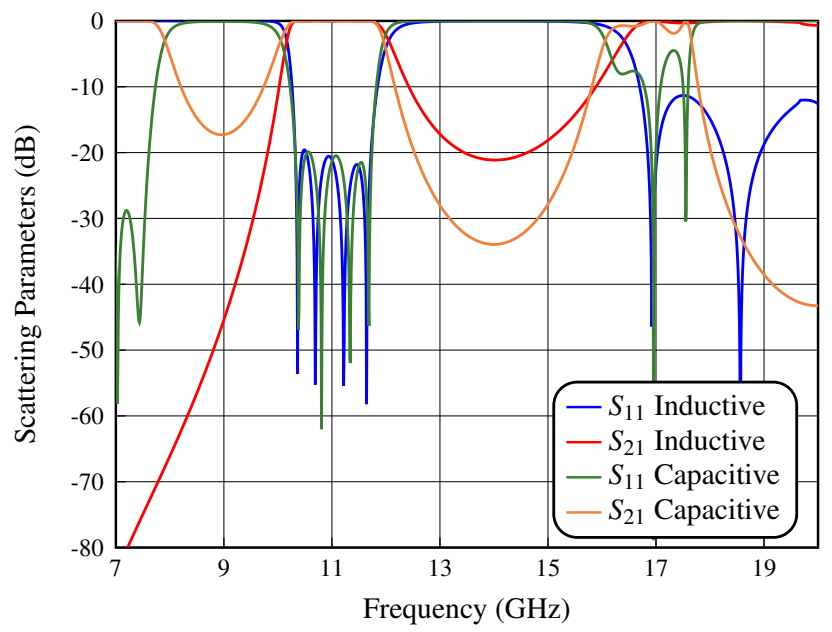

Fig. 2. Simulated performance of the inductive and capacitive filters.

Using capacitive irises (see Fig. 1, right), on the other hand, we can increase very significantly the attenuation of spurious signals at higher frequencies. Capacitive coupling irises, however, tend to have a rather small height for narrowband filter implementations. For wideband filter implementations, however, this is not an issue. Furthermore, the strength of the coupling can be adjusted using both the height and the thickness of the capacitive window. Capacitive windows can therefore be used also for relatively narrowband filters. A specific trade off between thickness and height must, however, be carried out in each specific case to identify the best configuration.

To better support our discussion, we show in Fig. 2 the comparison of the responses of two filters designed to satisfy the specifications given in section II. Both filters have been simulated using FEST3D (v.2018 by AuroraSAT, now with Dassault Systèmes). The difference between the two filters is that one uses only inductive couplings and the other only capacitive couplings (Fig. 3 and Fig. 4 show a 3D view of the two structures).

Looking more in detail at the difference between inductive and capacitive couplings, we note that there is one feature of inductive irises that is advantageous, namely, their lowfrequency behavior. Capacitive irises exhibit a resonant behavior below the filter pass band while inductive irises provide significant rejection. This is clearly shown in Fig. 2, where we can see that the capacitive filter provides no attenuation near the waveguide cut-off frequency. As expected, the inductive 


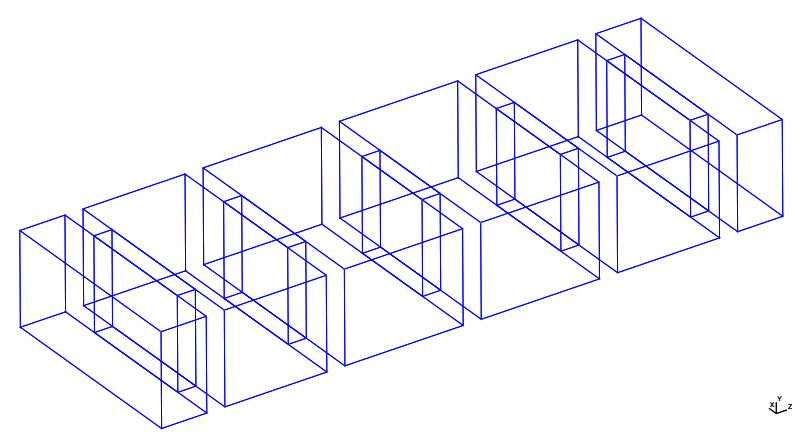

Fig. 3. Structure of the inductive filter.

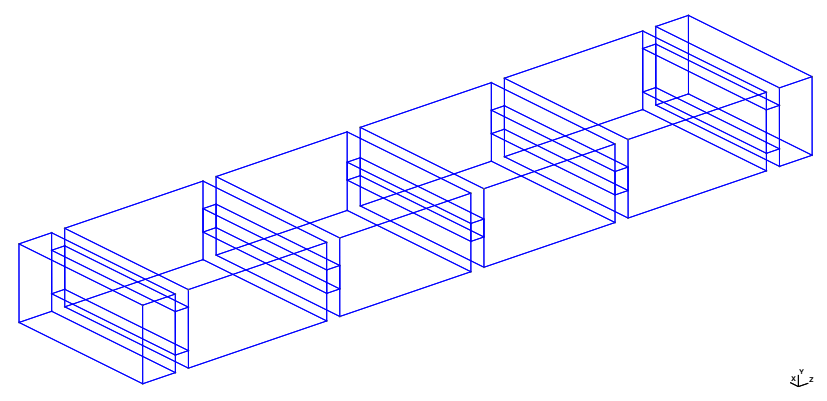

Fig. 4. Structure of the capacitive filter.

filter provides very poor response at higher frequencies.

Another issue that deserves more attention is that smaller coupling strengths are usually required in the central part of filter structures, whereas stronger couplings are usually required near the input and output ports.

In view of that, a possible solution to solve the limitations of both topologies (inductive and capacitive), is to mix inductive and capacitive irises along the structure of the filter. As a result, we will obtain the hybrid filter structure that is the main subject of this paper. The next step is then to investigate the relative positions of the inductive and capacitive irises to obtain the best overall filter performance.

To this end, we designed two 4-pole filters. The first with capacitive couplings but with an inductive iris in the center section (Geometry 1 in Fig. 5). The second, also with capacitive couplings, but with an inductive window in the first and last couplings (Geometry 2 in Fig. 6).

Fig. 7 shows a comparison of the simulated filter per-

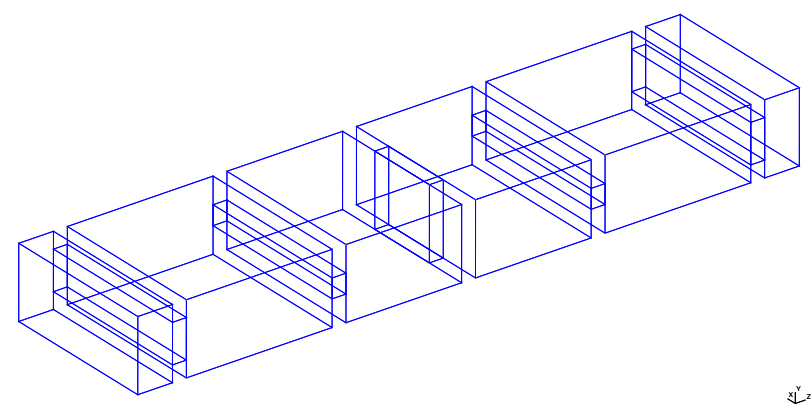

Fig. 5. Geometry 1 of the hybrid filter.

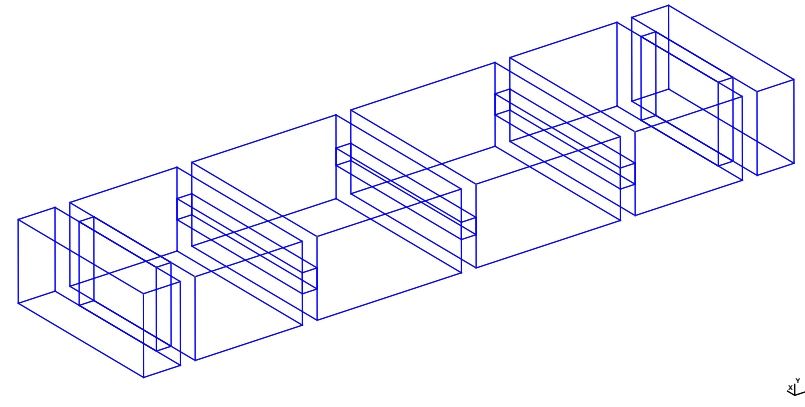

Fig. 6. Geometry 2 of the hybrid filter.

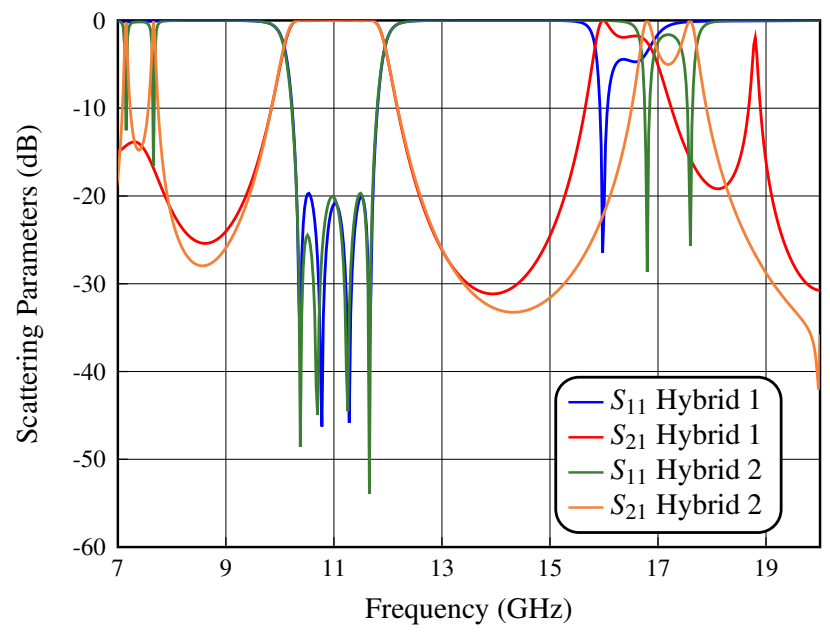

Fig. 7. Simulated performance of the hybrid 1 filter (Geometry 1) and hybrid 2 filter (Geometry 2).

formances. As we can see, the response of Geometry 2 shows an improvement in the attenuation of the first spurious signal of the filter. On the other hand, Geometry 1 shows an improvement response in the frequency range close to the cutoff frequency of the input waveguide, with a slight improvement in the attenuation of the spurious signal.

Based on the above discussion, we can therefore conclude this section by stating that the best solution is to use (a minimum of) inductive irises in the center of the filter. This choice will have a number of positive effects, namely, it will improve:

- Both the rejections of the filter in the lower and upper out-of-band frequency ranges.

- The manufacture of the filter since it will eliminate the need for capacitive windows with very small height.

- The footprint of the filter since inductively coupled resonators are, for the same resonant frequency, shorter than capacitively coupled ones.

Once the optimal position of the coupling windows and their type is found in a filter of order $N=4$, we can validate this solution with the design of two hybrid filters of order $N=8$. The filter 1 has three inductive irises in the center part of the filter, all other irises are capacitive. Filter 2 has four inductive irises (1st, 2nd, 8th and 9th) near the input/output and the rest are capacitive. The electrical performance of both filters is shown in Fig. 8. We can conclude that the use of a minimum 


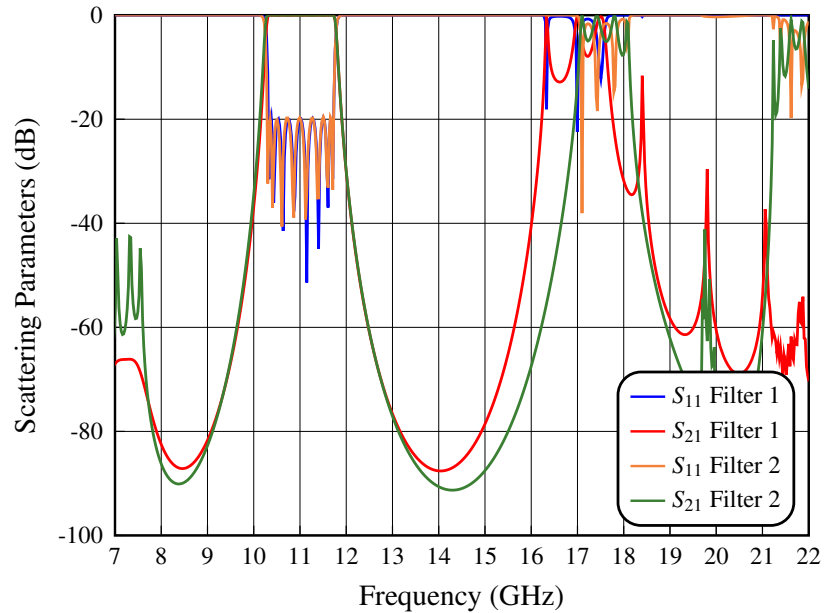

Fig. 8. Simulated performance of the filter 1 (three inductive irises in the center of the filter) and filter 2 (two inductive irises in the first and last coupling sections).

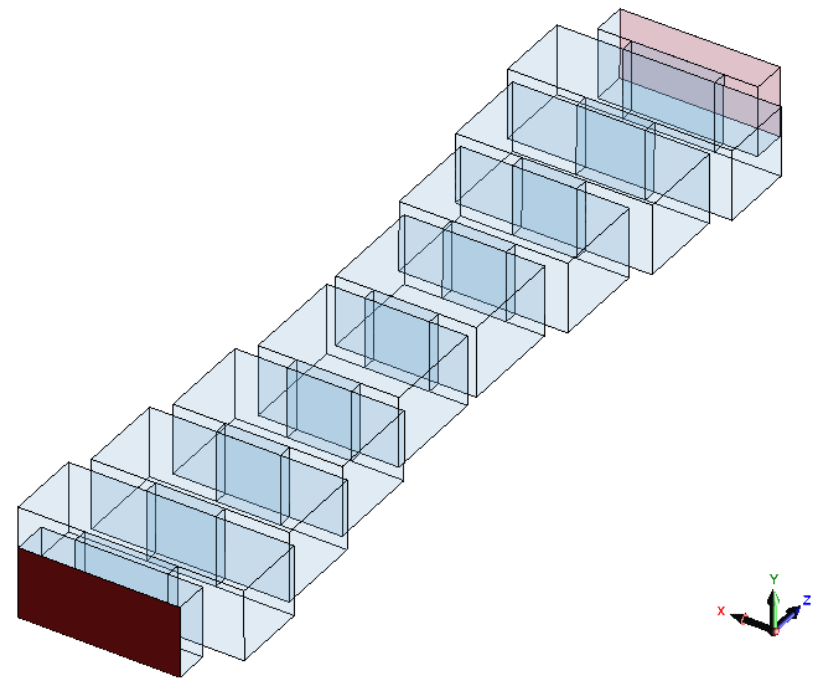

Fig. 9. Structure of the inductive filter 1.

number of inductive irises ( 3 in this case) in the center of the filter 1 shows major advantages when using both capacitive and inductive irises. Filter 1 has a higher rejection level than filter 2 in the lower rejection band.

\section{Changing Resonator Width}

As an alternative for the improvement of the out-of-band response of the classic inductive filter, we explore in this section the possibility of changing the width of the resonators. This is indeed a well-known approach, however, to the authors' knowledge, a detailed analysis of the possible advantages introduced has not been discussed in the technical literature. For a better understanding of this type of structure, and its effect on the performance of the filter, we designed two 8pole filters to satisfy the specifications in Table I. The first with widths that decrease as we approach the center of the structure (filter 1 in Fig. 9). The second with widths that increase as we approach the center of the structure (filter 2 in Fig. 10).

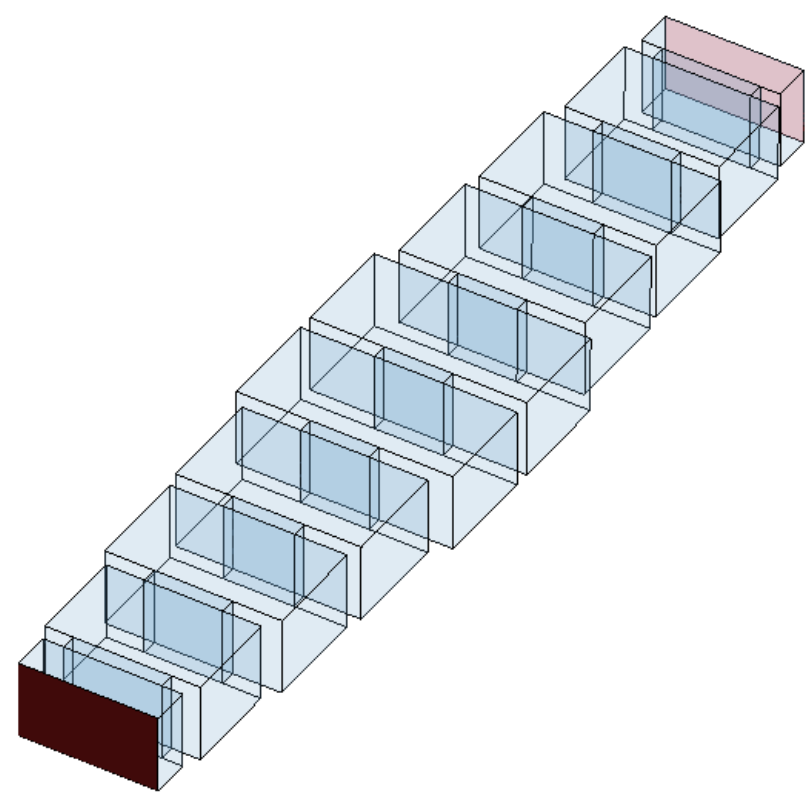

Fig. 10. Structure of the inductive filter 2 .

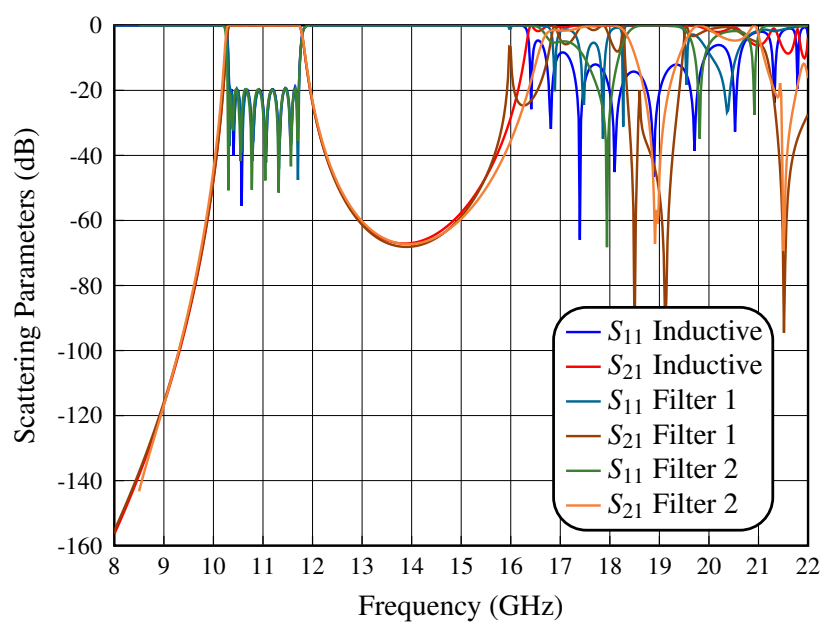

Fig. 11. Simulated performance of the inductive filter, filter 1 and filter 2 .

Fig. 11 shows a comparison of the simulated behavior of the structures of Fig. 9 and Fig. 10. The performances shown in Fig. 11 have been obtained using the commercial tool FEST3D. It is important to note that for the design of these structures the thickness of the irises has been kept constant.

As we can see, the responses of both inductive filters 1 and 2 show a slight improvement in the suppression of the first harmonic, in comparison with the classic inductive filter. However, both filter structures are longer.

To continue, we have designed also a capacitive filter with resonators of reduced width. Fig. 12 shows the structure of the filter applying the same width variation as the inductive filter in Fig. 9. The resulting slight improvement of the out-ofband response can be appreciated in Fig. 13. We can therefore conclude that the best topology is to use resonators with decreasing widths towards the center of structure. 


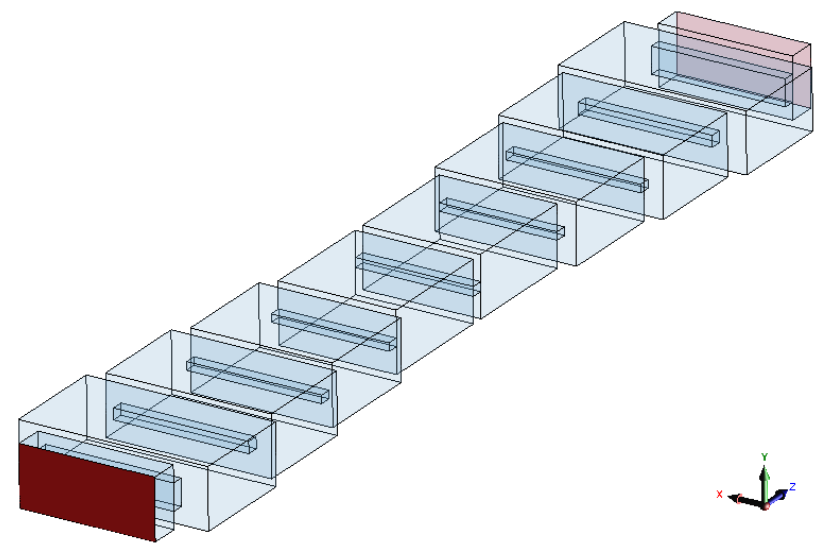

Fig. 12. Structure of the capacitive filter with resonators of different width (filter 3).

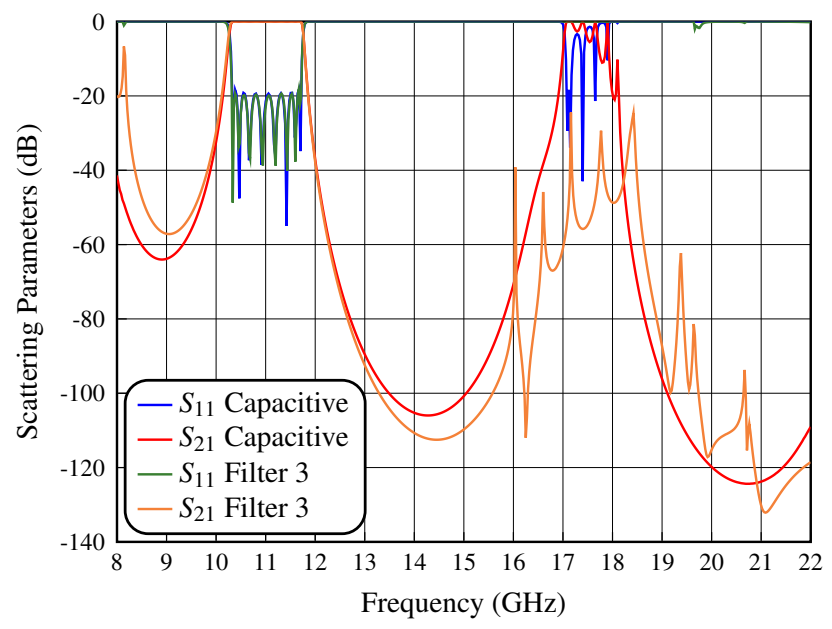

Fig. 13. Simulated performance of the capacitive filter and filter 3 .

\section{DESIGN OF A CAPACITIVE SIR FILTER}

The inductive and capacitive topologies discussed in the previous sections, were built with uniform impedance resonators (UIRs). A significant improvement of the out-of-band performance can be obtained using SIRs, as it was proposed for rectangular waveguide filters in [46].

A further improvement of the out-of-band response of the filter can be obtained by using SIRs in conjunction with capacitive couplings, as shown in Fig. 14. Another advantage introduced by this structure is the reduction of the total length

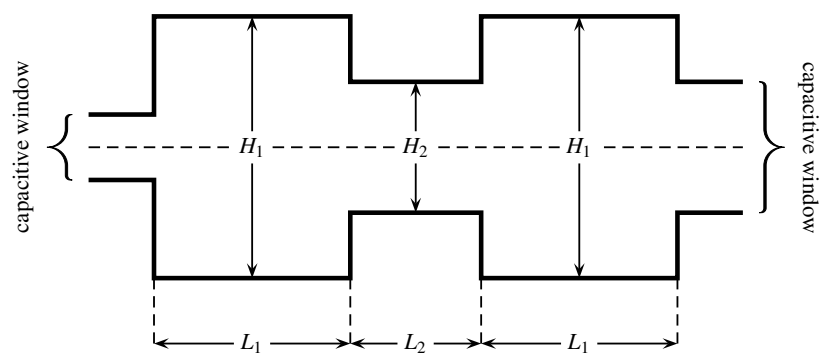

Fig. 14. Geometry of a basic SIR with capacitive input and output couplings (side view).

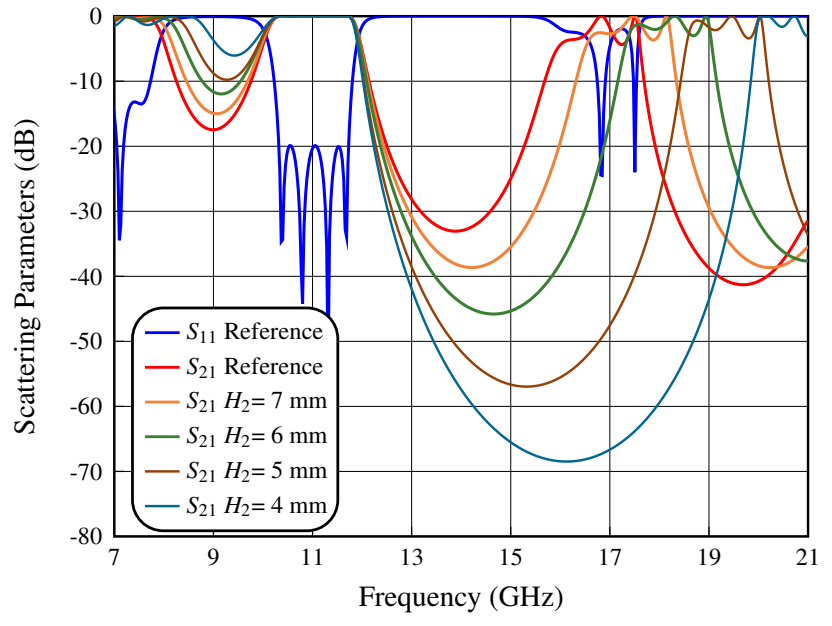

Fig. 15. Simulated performance of SIR implementations in a capacitive filter.

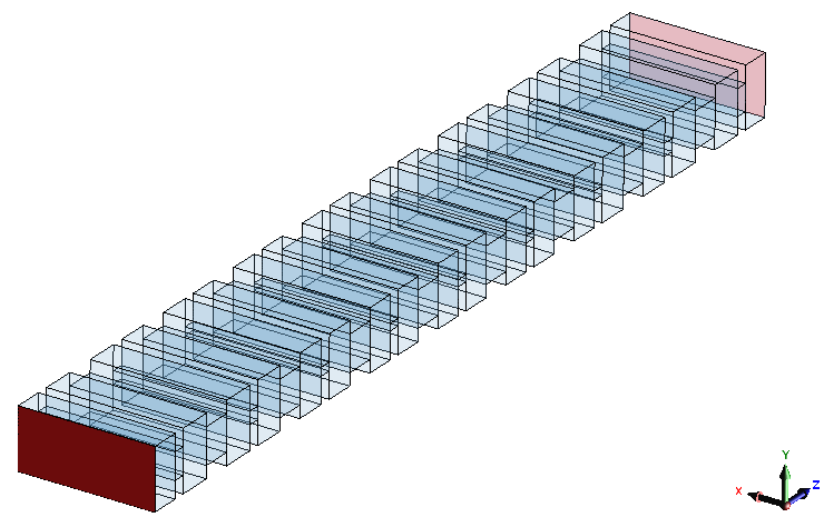

Fig. 16. Structure of the capacitive filter with SIR resonators.

of each resonator, due to the reduced height of the central section.

It is important to mention that the geometrical parameters of the SIR ( $H_{1}, H_{2}, L_{1}$ and $L_{2}$ in Fig. 14) in our study, have been optimized with a full-wave EM simulation tool in order to obtain a good rejection level and a spurious-free range that is as large as possible. In the optimization process we have considered $H_{1}=8.0 \mathrm{~mm}, L_{2}=5.0 \mathrm{~mm}$, and different values of $\mathrm{H}_{2}$ (see Fig. 15).

In Fig. 15, we can see that for $H_{2}=4.0 \mathrm{~mm}$, the response of the capacitive SIR filter (light blue line) is significantly better than the one of the UIR filter (red line). We therefore used $H_{2}=4.0 \mathrm{~mm}$, and $L_{2}=5.0 \mathrm{~mm}$ in the SIRs to design an 8-pole capacitive filter, as shown in Fig. 16. The dimension of $L_{1}$ for all SIRs, on the other hand, is defined during the filter design process.

In Fig. 17 we now show the comparison between the responses of a capacitive filter designed with traditional resonators (UIR), another with different widths (filter 3 in section IV), and the SIR filter with capacitive couplings. As we can see in Fig. 17, using SIRs, the spurious signals are attenuated and appear further away from the pass band. However, we can also see a slight increase in the $S_{21}$ response of the filter below the pass band. This feature could be 


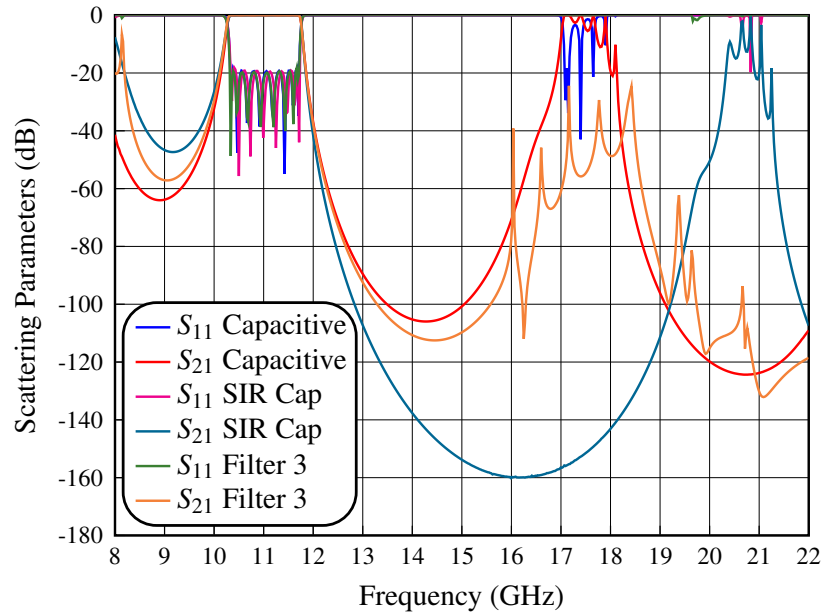

Fig. 17. Simulated performance of the capacitive UIR filter, filter 3 and capacitive SIR filter.

improved with the use of inductive irises. In any case, the out-of-band response of the filter with SIRs is much better than the one of the filter with changes in the width of the resonators.

In conclusion, using both techniques, namely, mixed coupling (inductive and capacitive) and SIRs, we should be able to obtain a wide spurious-free region, with an acceptable rejection level below the pass band. In the next section, we will discuss in detail the design procedure of the hybrid SIR filter, initially discussed in [48], with special emphasis on the use of the Aggressive Space Mapping (ASM) technique.

\section{Wideband Filter Design}

A Chebyshev filter of order 8, centered at $11 \mathrm{GHz}$, with a bandwidth of $1410 \mathrm{MHz}$, and WR-90 input and output waveguide, is designed with three central inductive windows. As we have described in section III, the advantage of using three inductive irises in the center of the filter is in fact due to the higher rejection achieved below the pass band.

All other coupling windows are capacitive. All resonators are SIR, with the $H_{2}$ and $L_{2}$ values obtained in section V (i.e. $H_{2}=4.0 \mathrm{~mm}$, and $L_{2}=5.0 \mathrm{~mm}$ ). The other dimensions of the SIRs will be obtained using an ASM-based optimization procedure outlined next. In Fig. 18 we show the resulting filter structure.

It is important to remember that the central heights of the SIRs have been fixed to $H_{2}=4.0 \mathrm{~mm}$ in order to obtain a spurious-free range above the pass band that is as large as possible. This value is also appropriate to ensure that there are no negative high power effects. The other critical elements in terms of power effects are the capacitive irises. A trade off has therefore been carried out in the design process to ensure that iris heights (gaps) are appropriate for the intended power levels.

The complete procedure for designing these filters is composed of the following steps:

1) Design the waveguide filter (coupling elements and resonators) following the procedure outlined in section II, considering the practical recommendations given next.

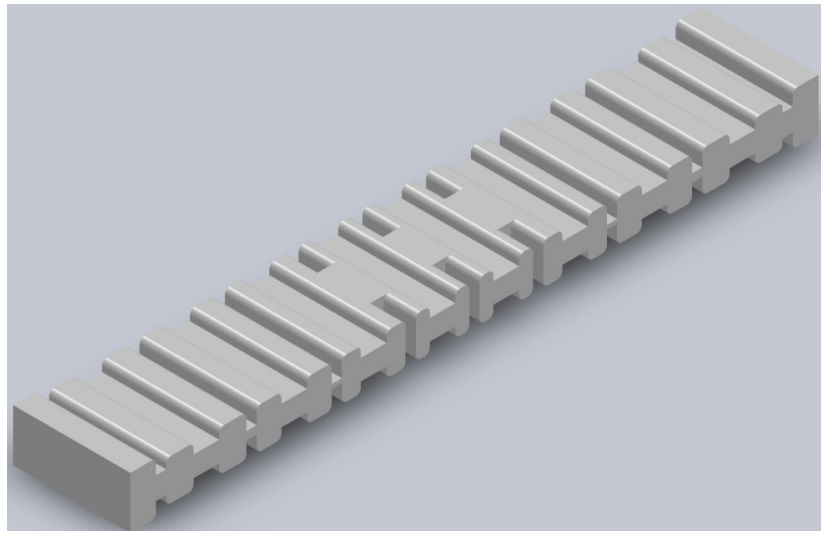

Fig. 18. Structure of the hybrid SIR filter.

2) The number of inductive coupling windows of the filters are selected empirically. For practical orders $(N \geq 4)$ our study gives $n=\lceil N / 2-1\rceil$, where $n$ is the number of inductive windows to be located in the inner central sections of the filter, and $N$ is the filter order.

3) All resonators should be SIRs to obtain the best out-ofband performance. The total length see Fig. 14 is around $\lambda / 2$ (where $\lambda$ is the guide wavelength evaluated at the center frequency). The central section length is chosen to be approximately $L_{2}=\lambda / 6$. The remaining dimensions $\left(H_{1}\right.$ and $\left.H_{2}\right)$ are obtained following the guidelines of section V.

The design of the structure has been carried out in two steps, using a combination of the procedure described in [50], and the aggressive spacing mapping (ASM) technique [51]. In particular, the procedure described in [50] has been used to obtain the initial dimensions of the filter structure, while the ASM has been used for the final optimization stage. As it is well known, the ASM method is based on the use of two electromagnetic simulators. In our case, the first one is FEST3D, which can be used in a low accuracy setting that is computationally very efficient. The second simulator we used is CST Design Studio (v.2018, CST GmbH, now with Dassault Systèmes) which is highly accurate, but is computationally less efficient.

In the first step of the design process, we designed a filter structure with sharp corners (90 degrees). In the second design step we have changed the sharp corners into rounded corners $(r=1.0 \mathrm{~mm})$, in order to reduce the manufacturing cost by including Computer Numerical Control (CNC) machining features into the design cycle. The final values for all dimensions of the hybrid SIR filter are collected in Table II. The dimensions are given up to the center of the filter because the filter is symmetrical. The thickness of the windows is $t$, and the SIRs have the same width as the input waveguide. The other variables are indicated in Fig. 1 and Fig. 14.

A prototype of the filter has been manufactured in a clamshell configuration using a combination of milling and spark erosion (see Fig. 19). The comparison between measured and simulated in-band and out-of-band responses are shown in Fig. 20 and 21, respectively. 


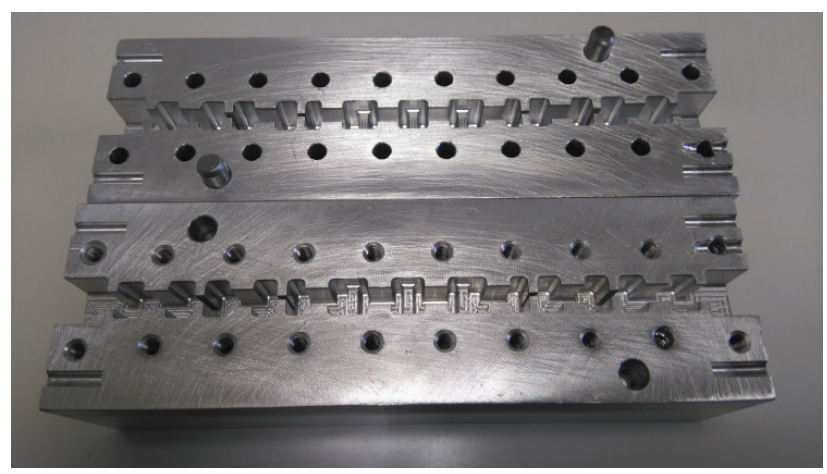

Fig. 19. Manufactured prototype in aluminum (no silver plating).

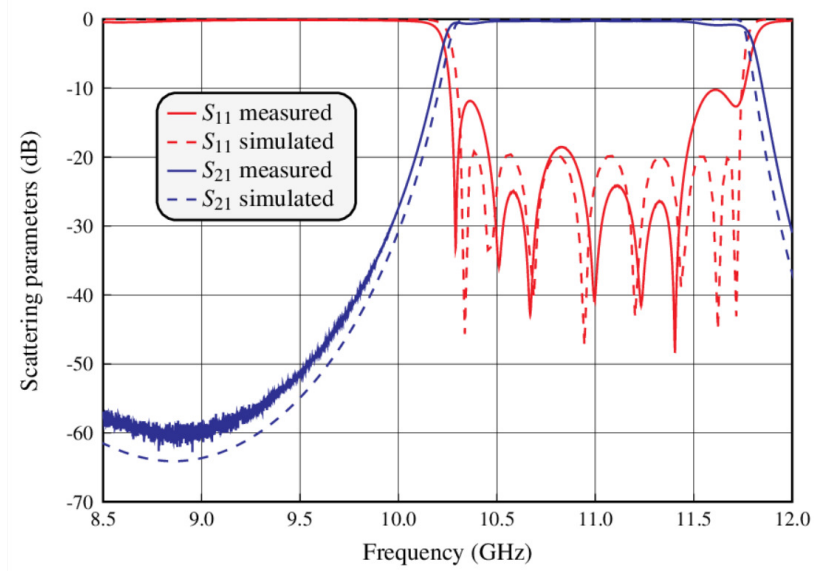

Fig. 20. Measurement of the in-band performance of the filter compared with the EM simulation.

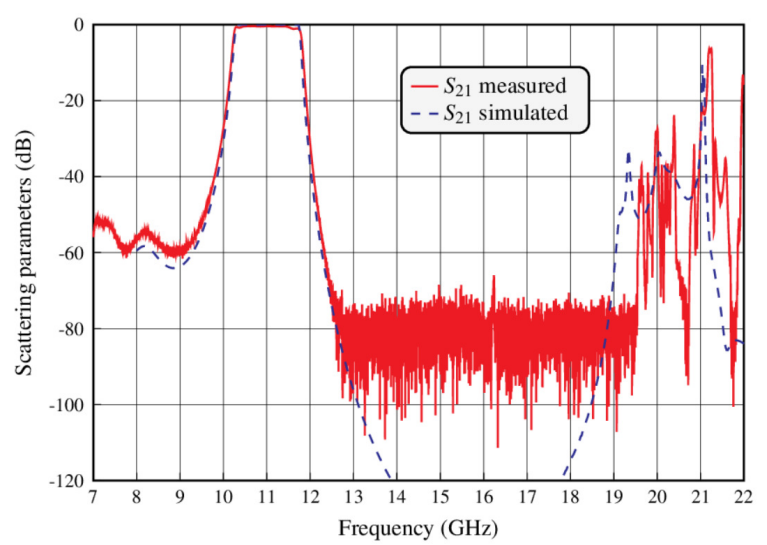

Fig. 21. Measurement of the out-of-band performance of the filter compared with the EM simulation.
TABLE II

PHYSICAL DIMENSIONS FOR THE IN-LINE HYBRID SIR FILTER.

\begin{tabular}{|c|c|}
\hline Section Type & Dimensions $(\mathrm{mm})$ \\
\hline Input Waveguide & $\begin{array}{l}a=22.86 \\
b=10.16\end{array}$ \\
\hline Capacitive window 1 & $\begin{array}{l}a=22.86 \\
h=3.772 \\
t=2\end{array}$ \\
\hline SIR 1 & $\begin{array}{l}H_{1}=8.000 L_{1}=5.632 \\
H_{2}=4.000 L_{2}=5.000\end{array}$ \\
\hline Capacitive window 2 & $\begin{array}{l}a=22.86 \\
h=1.476 \\
t=2\end{array}$ \\
\hline SIR 2 & $\begin{array}{l}H_{1}=8.000 L_{1}=5.025 \\
H_{2}=4.000 L_{2}=5.000\end{array}$ \\
\hline Capacitive window 3 & $\begin{array}{l}a=22.86 \\
h=0.805 \\
t=2\end{array}$ \\
\hline SIR 3 & $\begin{array}{l}H_{1}=8.000 L_{1}=3.771 \\
H_{2}=4.000 L_{2}=5.000\end{array}$ \\
\hline Inductive window 1 & $\begin{array}{l}w=9.363 \\
b=8.000 \\
t=2\end{array}$ \\
\hline SIR 4 & $\begin{array}{l}H_{1}=8.000 L_{1}=2.576 \\
H_{2}=4.000 L_{2}=5.000\end{array}$ \\
\hline Inductive window 2 & $\begin{array}{l}w=8.546 \\
b=8.000 \\
t=2\end{array}$ \\
\hline
\end{tabular}

As we can see, although the in-band performance is indeed centered at $11 \mathrm{GHz}$, the return loss is degraded with respect to the simulations. Furthermore, there is a slight disagreement between simulations and measurements in the out-of-band performance, namely, a small shift in the frequency of the first spurious response. Nevertheless, the agreement between simulations and measurements is good enough to validate both the filter structure and the design procedure. The discrepancies are fully justified by the limited accuracy of the manufacturing process (the manufacturing tolerance was estimated to be about 60 microns). This point will be further confirmed with a sensitivity analysis, as detailed in section IX.

\section{SIR FILTER WITH BODY AND COVER}

To further improve the filter response, and try to avoid the issues related to manufacturing errors, we have next designed a hybrid SIR filter with a number of tuning elements in the SIR resonators. It is important to mention that the geometry of the filter has been modified in order to include the tuning elements. The modification consisted in introducing a vertical offset in all the elements of the filter, so that the structure could be built with a flat top cover. The tuning elements have then been fastened to the flat top cover. The first step in this new design is, therefore, to study the effect of introducing an offset in the position of the capacitive irises, as show in Fig. 22. Fig. 23 shows the comparison between the responses of the capacitive filter with centered irises (filter 1), and offset irises 


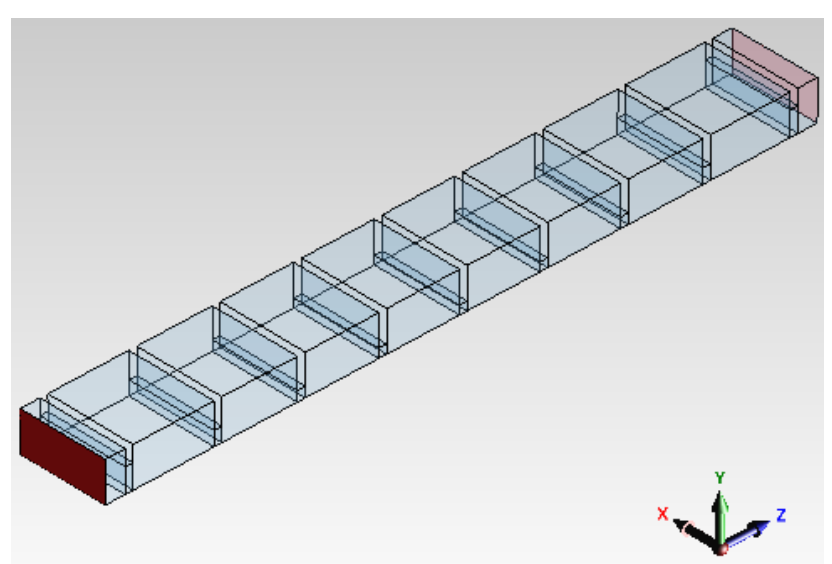

Fig. 22. Capacitive filter with offset irises (close to the cover).

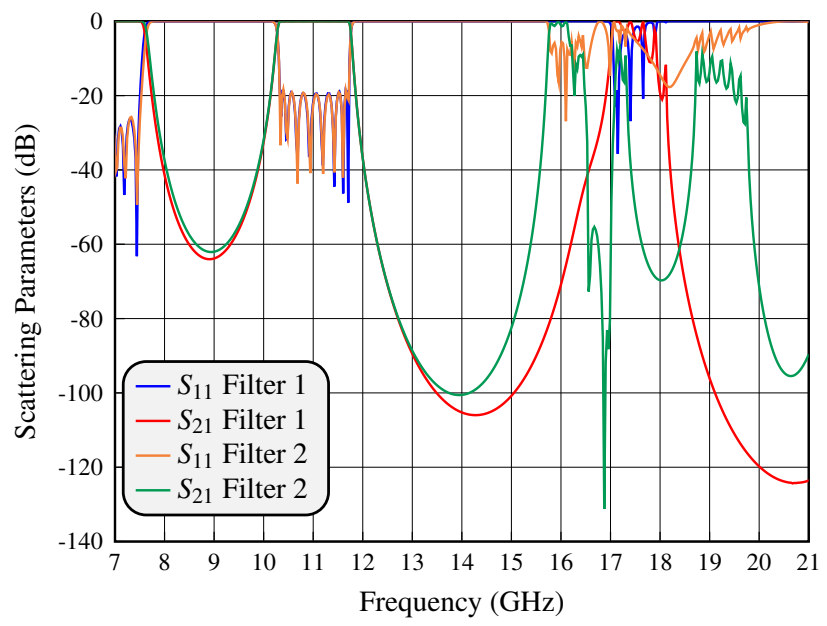

Fig. 23. Simulated performance of the capacitive filter 1 and filter 2 .

(filter 2). As we can see, the change resulted in a reduction of the frequency location of the first spurious, and an increase in the height of capacitive irises.

Next, the in-line hybrid SIR filter was redesigned, obtaining the structure shown in Fig. 24. The comparison between the responses of the hybrid SIR filter (see Fig. 18) with centered irises (filter 3) and with offset irises (filter 4) is shown in Fig. 25. As we can see, changing the position of capacitive irises improves the out-of-band response by reducing the level of the first spurious response.

The next step in the design has been to change the offset of the SIR sections. In this context, we show in Fig. 26 a hybrid filter with offsets in both irises and SIRs. The comparison between the hybrid SIR with centered SIR sections (filter 4), and hybrid SIR with offsets (filter 5) is shown in Fig. 27.

As we can see, using an offset in both irises and SIR sections improves the out-of-band response, increases the height of the capacitive irises (that is the gap between metallic surfaces), and reduces the manufacturing costs.

An additional benefit of the offset configuration is that it allows the introduction of tuning elements of radius $r=0.9$ $\mathrm{mm}$ in the central section of the SIR resonators to compensate manufacturing errors, and further reduce manufacturing costs.

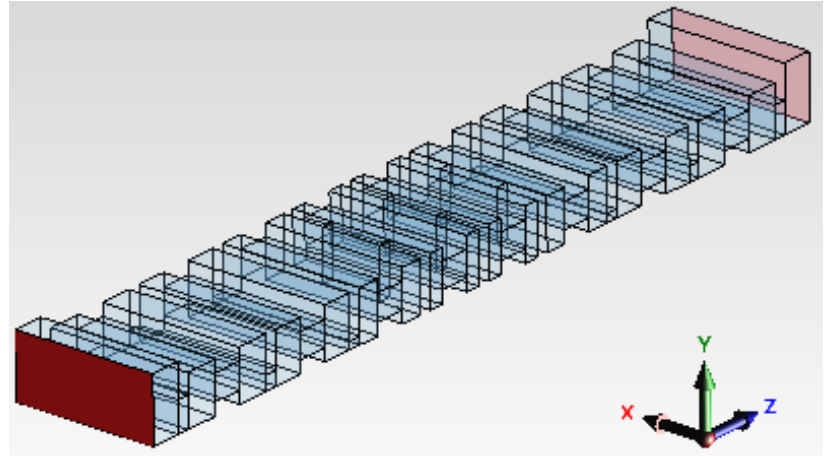

Fig. 24. Hybrid SIR filter with offset iris (close to the cover).

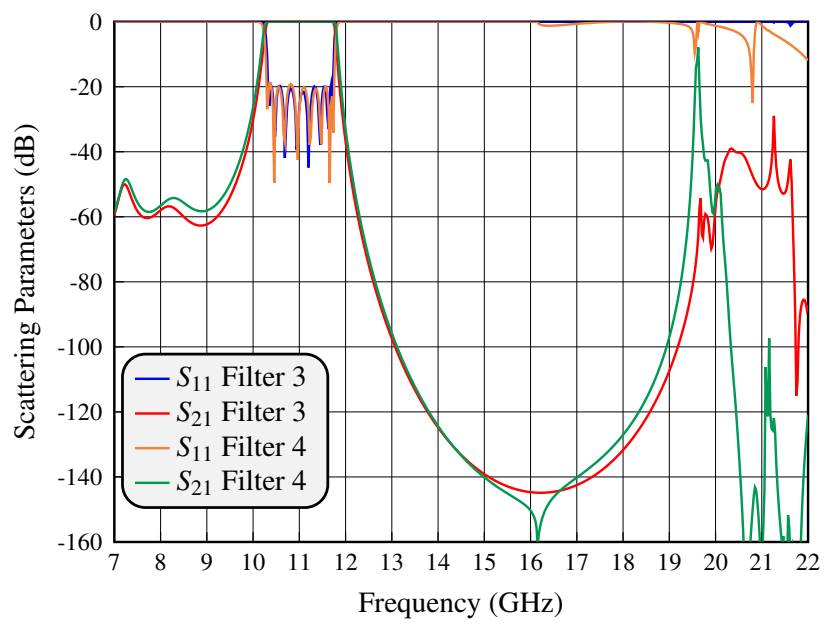

Fig. 25. Simulated performance of the hybrid SIR filter 3 and filter 4 .

In Fig. 28 we show the final geometry of the hybrid SIR filter with a body (where irises and SIR sections are implemented) and a cover (including tuning elements), named as filter 6 . It is important to note, at this point, that tuning elements cannot be used in the capacitive coupling irises due to the limited space available.

The comparison between the responses of filters with and without tuning elements is shown in Fig. 29. As we can see, when using tuning elements the response of the filter does not change, but the level of spurious is slightly higher.

The prototype body and cover have been manufactured

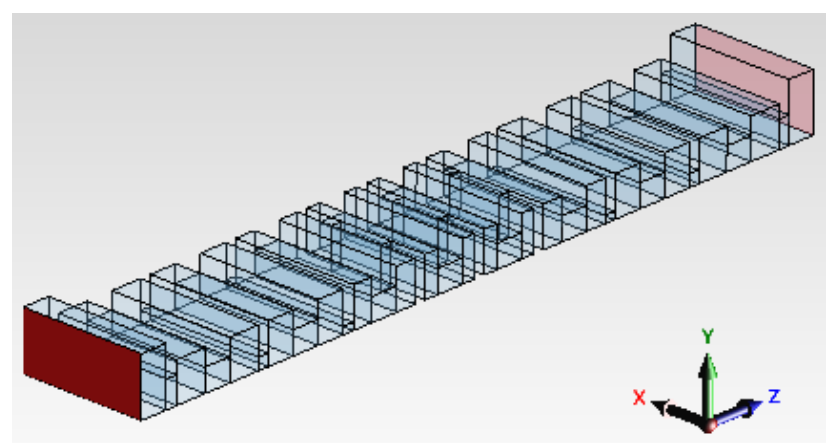

Fig. 26. Hybrid SIR filter with offset irises and resonators to be implemented within a body (to be closed with a flat top cover). 


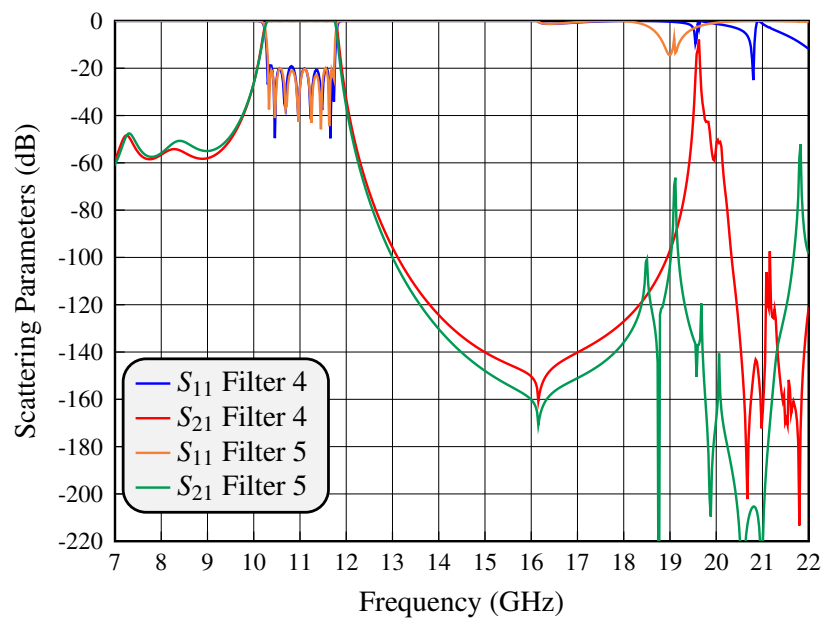

Fig. 27. Simulated performance of the hybrid SIR filter 4 and filter 5 .

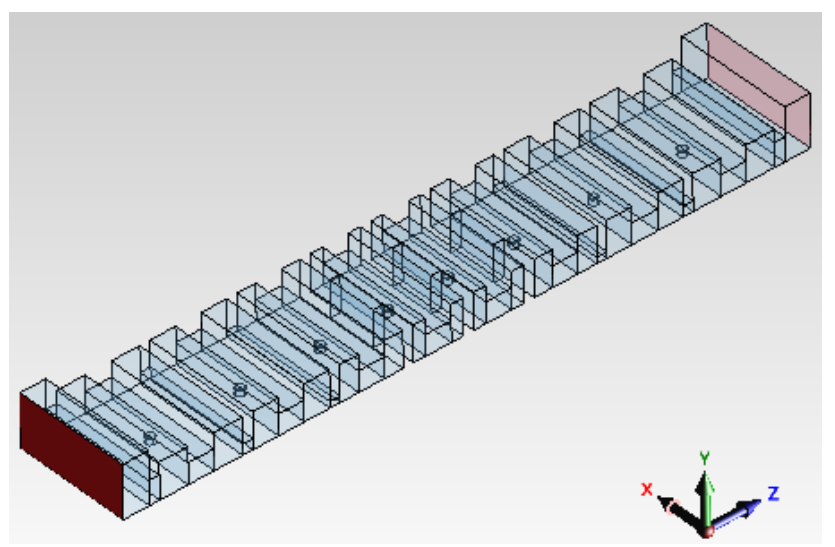

Fig. 28. Hybrid SIR filter manufactured in two parts: body and cover. Tuning screws are also included in the flat cover.

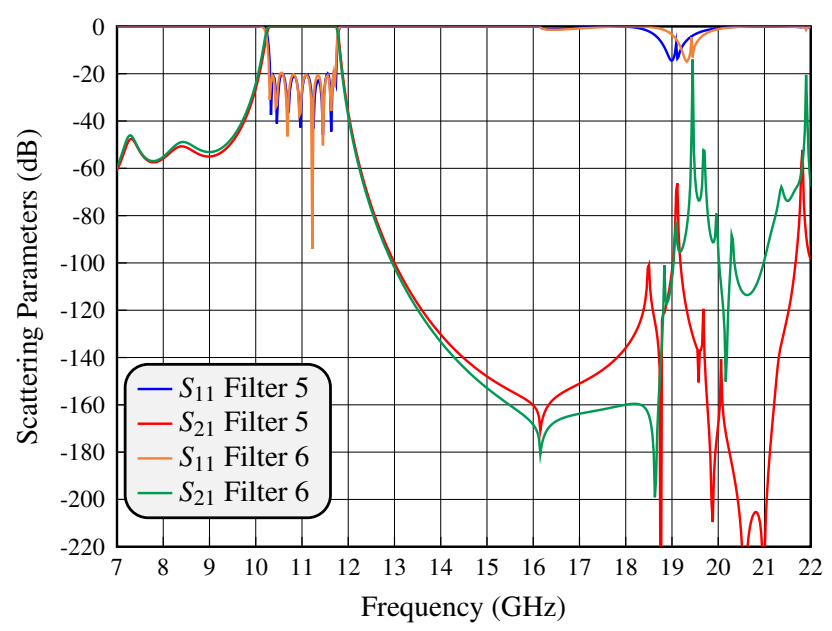

Fig. 29. Simulated performance of the hybrid SIR filter 5 and filter 6 .

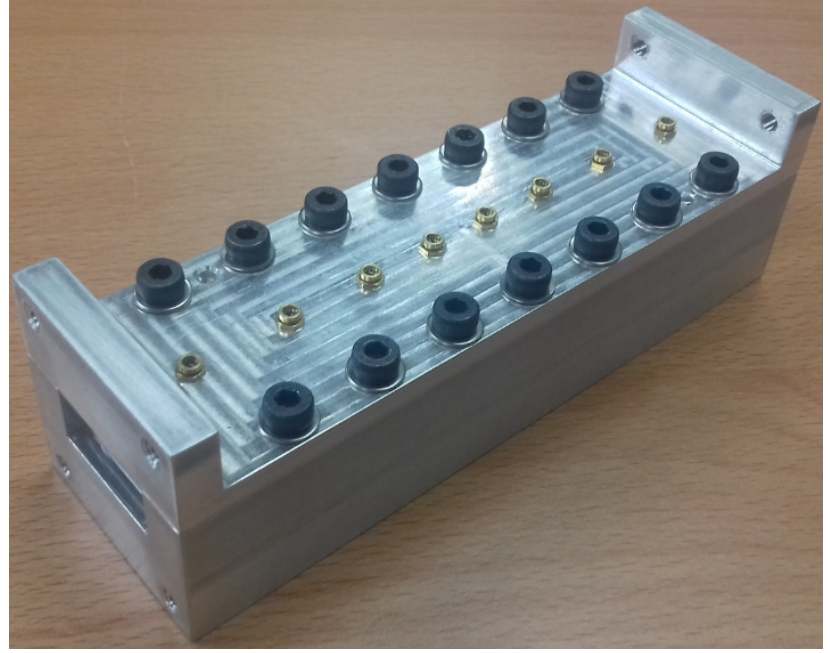

Fig. 30. Manufactured prototype (body and cover) in aluminum (no silver plating).

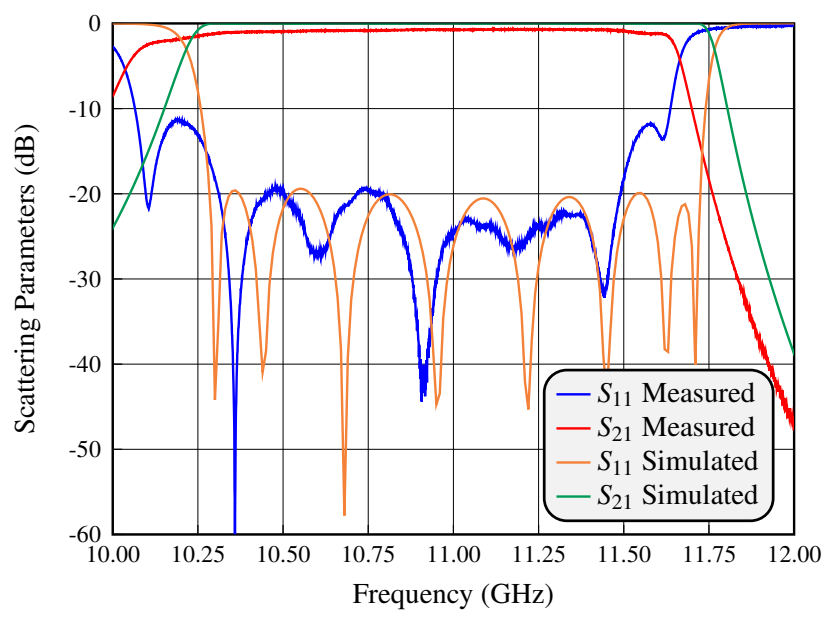

Fig. 31. Measurement of the in-band performance of the filter with body and cover compared with the EM simulation.

using standard milling (see Fig. 30). The comparison between measured and simulated in-band and out-of-band responses are shown in Fig. 31 and 32, respectively.

Table III shows the physical dimensions for the in-line hybrid SIR filter with body and cover. The dimensions are given up to the center of the filter because the filter is symmetrical. The thickness of the windows is $t$, and the SIRs have the same width as the input waveguide.

As we can see, although the in-band performance is indeed centered at $11 \mathrm{GHz}$, the return loss is degraded with respect to the simulations, and it shows a small shift in the center frequency of the pass band, even though the filter now has eight tuning elements. Also in the out-of-band response, there is a slight disagreement between simulations and measurements. In conclusion, using this new structure with tuning elements can result in a viable lower cost implementation. However, it is apparent that the problems caused by the low manufacturing accuracy cannot be completely eliminated using only tunable SIR sections. 


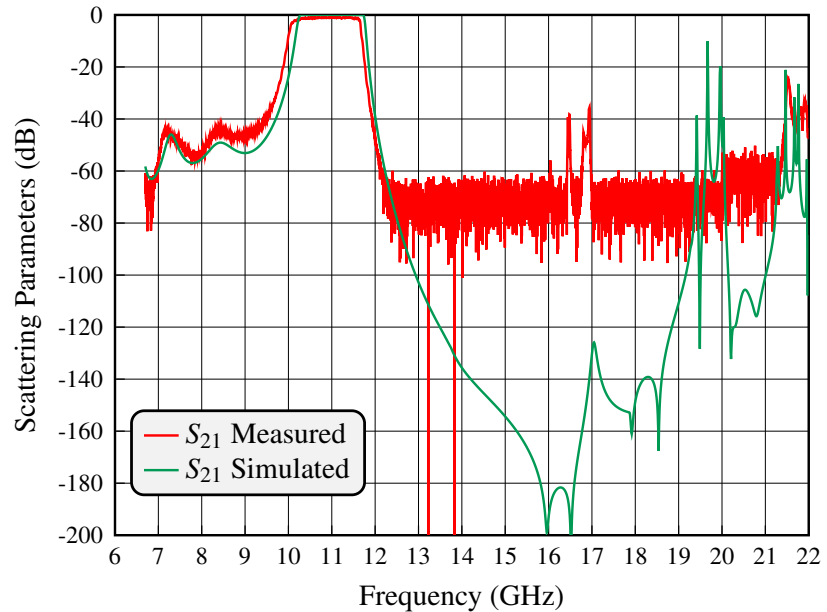

Fig. 32. Measurement of the out-of-band performance of the filter with body and cover compared with the EM simulation.

TABLE III

PHYSICAL DIMENSIONS FOR THE HYBRID SIR FILTER WITH BODY AND COVER.

\begin{tabular}{|c|c|}
\hline Section Type & Dimensions $(\mathrm{mm})$ \\
\hline Input Waveguide & $\begin{array}{l}a=22.86 \\
b=10.16\end{array}$ \\
\hline Capacitive window 1 & $\begin{array}{l}a=22.86 \\
h=4.734 \\
t=2\end{array}$ \\
\hline SIR 1 & $\begin{array}{l}H_{1}=7.000 L_{1}=5.485 \\
H_{2}=4.000 L_{2}=5.000\end{array}$ \\
\hline Capacitive window 2 & $\begin{array}{l}a=22.86 \\
h=2.028 \\
t=2\end{array}$ \\
\hline SIR 2 & $\begin{array}{l}H_{1}=7.000 L_{1}=4.943 \\
H_{2}=4.000 L_{2}=5.000\end{array}$ \\
\hline Capacitive window 3 & $\begin{array}{l}a=22.86 \\
h=1.199 \\
t=2\end{array}$ \\
\hline SIR 3 & $\begin{array}{l}H_{1}=7.000 L_{1}=3.624 \\
H_{2}=4.000 L_{2}=5.000\end{array}$ \\
\hline Inductive window 1 & $\begin{array}{l}w=9.541 \\
b=7.000 \\
t=2\end{array}$ \\
\hline SIR 4 & $\begin{array}{l}H_{1}=7.000 L_{1}=2.382 \\
H_{2}=4.000 L_{2}=5.000\end{array}$ \\
\hline Inductive window 2 & $\begin{array}{l}w=8.616 \\
b=7.000 \\
t=2\end{array}$ \\
\hline
\end{tabular}

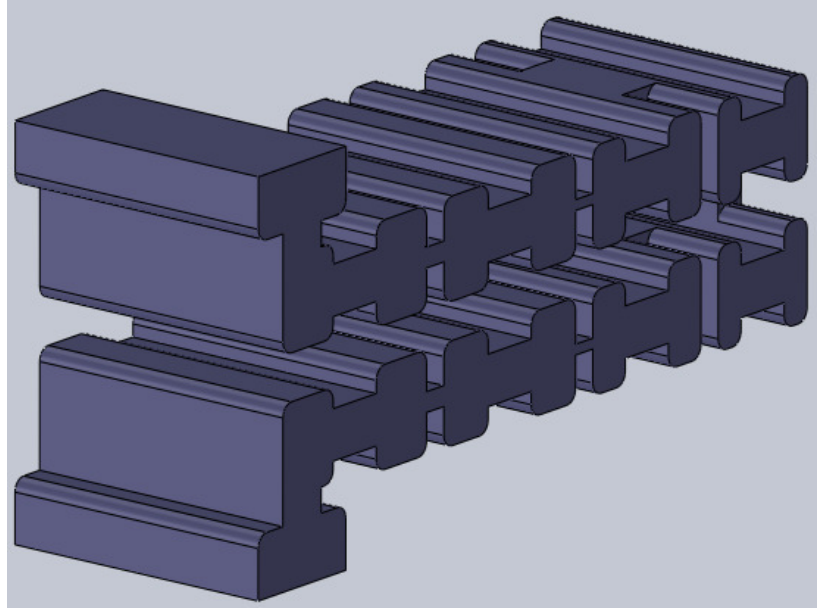

Fig. 33. E-plane rounded hybrid SIR filter.

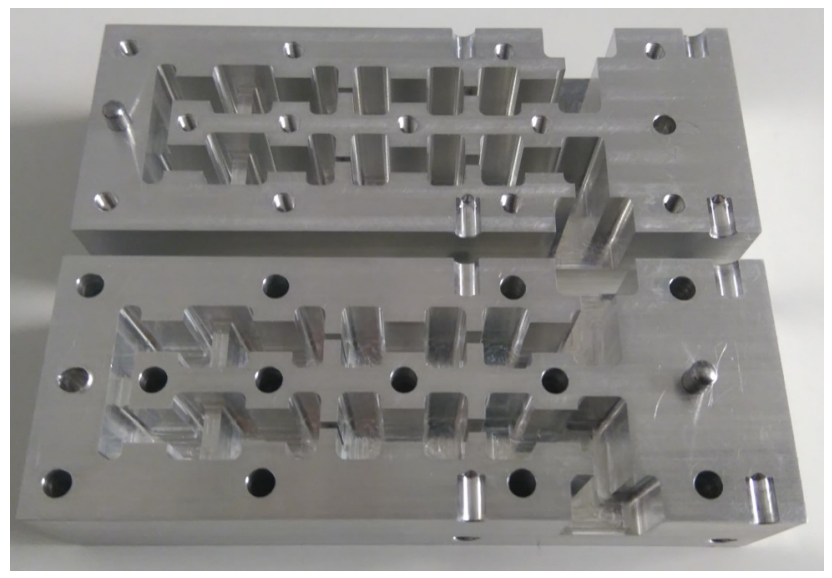

Fig. 34. Manufactured prototype in aluminum (no silver plating).

\section{COMPACT FILTER DESIGN}

The final filter design that we discuss in this paper is a folded implementation of the basic hybrid SIR filter. The first step in the design process is to fold the structure as shown in Fig. 33. One important detail is that for the central inductive iris we have chosen a thickness of $3.0 \mathrm{~mm}$, in order to avoid mechanical problems. Table IV shows the detailed physical dimensions for the E-plane folded hybrid SIR filter.

A further important detail is that we must ensure that the filter can be properly connected to the input and output waveguide. For this reason we have introduced in the structure two more 90 degrees bends in the routing. Again, the final filter optimization has been performed using the ASM procedure, including also the effects of rounded corners (with $r=1.0$ mm, see Fig. 33).

The new folded prototype has been manufactured in a clamshell configuration using a combination of milling and spark erosion (see Fig. 34). In this case, however, the manufacturing error has been kept below 10 microns for all the curved corners, and below 5 microns for all other filter elements. The comparison between measured and simulated in-band and outof-band responses are shown in Fig. 35 and 36, respectively. 
TABLE IV

PHYSICAL DIMENSIONS FOR THE E-PLANE FOLDED HYBRID SIR FILTER.

\begin{tabular}{|c|c|}
\hline Section Type & Dimensions (mm) \\
\hline Input Waveguide & $\begin{array}{l}a=22.86 \\
b=10.16\end{array}$ \\
\hline Capacitive window 1 & $\begin{array}{l}a=22.86 \\
h=4.445 \\
t=2\end{array}$ \\
\hline SIR 1 & $\begin{array}{l}H_{1}=7.271 \quad L_{1}=5.828 \\
H_{2}=4.000 L_{2}=5.000 \\
H_{1}=8.000 L_{1}=5.834\end{array}$ \\
\hline Capacitive window 2 & $\begin{array}{l}a=22.86 \\
h=1.591 \\
t=2\end{array}$ \\
\hline SIR 2 & $\begin{array}{l}H_{1}=8.000 L_{1}=5.138 \\
H_{2}=4.000 L_{2}=5.000\end{array}$ \\
\hline Capacitive window 3 & $\begin{array}{l}a=22.86 \\
h=0.830 \\
t=2\end{array}$ \\
\hline SIR 3 & $\begin{array}{l}H_{1}=8.000 L_{1}=3.771 \\
H_{2}=4.000 L_{2}=5.000\end{array}$ \\
\hline Inductive window 1 & $\begin{array}{l}w=9.617 \\
b=8.000 \\
t=2\end{array}$ \\
\hline SIR 4 & $\begin{aligned} H_{1} & =8.000 L_{1}=2.657 \\
H_{2} & =4.000 L_{2}=5.000\end{aligned}$ \\
\hline Inductive window 2 & $\begin{array}{l}w=10.442 \\
b=2.657 \\
t=3\end{array}$ \\
\hline
\end{tabular}

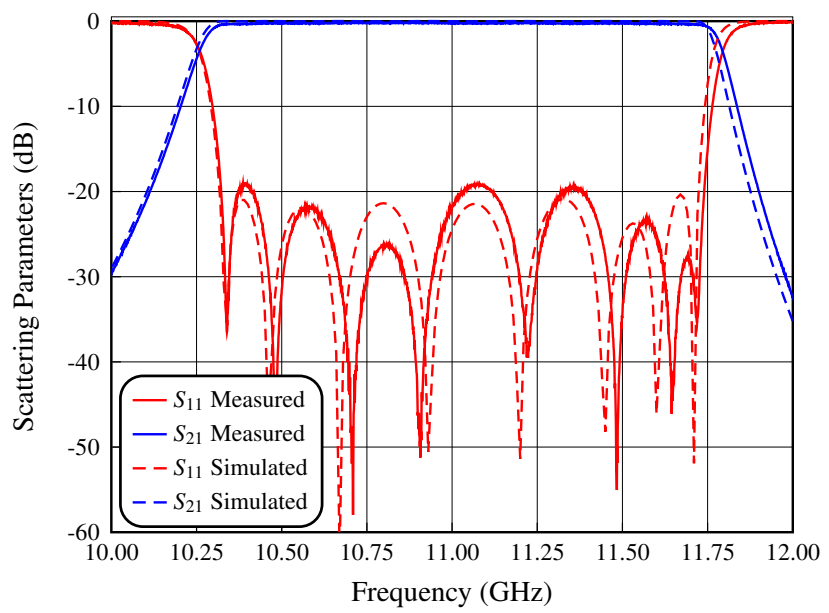

Fig. 35. Measurement of the in-band performance of the folded E-plane filter compared with the EM simulation.

As we can see, the in-band performance is indeed centered at $11 \mathrm{GHz}$, the 8 poles of the filter are clearly visible, and the agreement with the simulation is now excellent. There is also a good agreement between simulation and measurement in the out-of-band response. However, there is a spike reaching $-55 \mathrm{~dB}$ of rejection at about $16.76 \mathrm{GHz}$. The effects of the increased quality of the manufacturing process are clearly evident, even though the effects of errors can not be com-

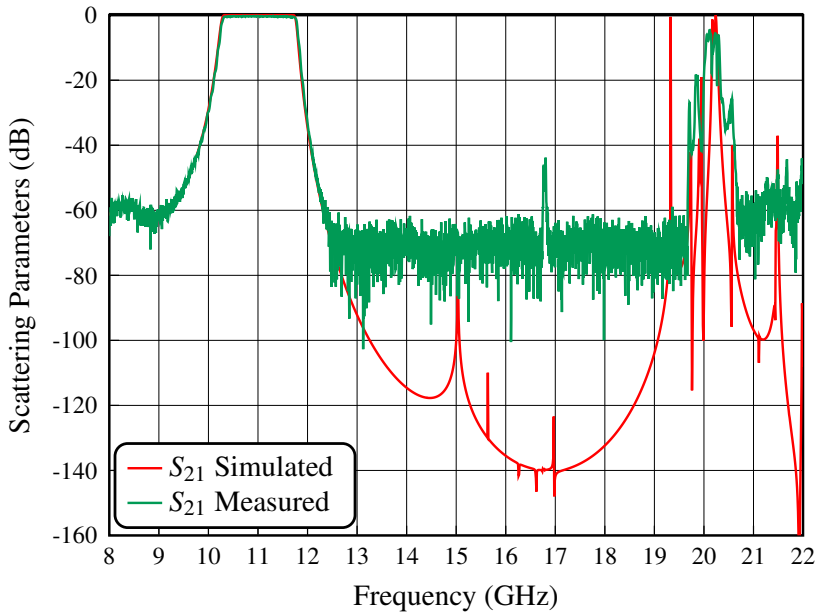

Fig. 36. Measurement of the out-of-band performance of the folded E-plane filter compared with the EM simulation.

TABLE V

TOLERANCE ANALYSIS OF THE FABRICATED STRUCTURES.

\begin{tabular}{|c|c|c|c|c|c|}
\hline Filter Type & \multicolumn{5}{|c|}{ Yield of the Structures } \\
\hline \hline Tolerance & $5 \mu \mathrm{m}$ & $10 \mu \mathrm{m}$ & $15 \mu \mathrm{m}$ & $25 \mu \mathrm{m}$ & $40 \mu \mathrm{m}$ \\
\hline In-line Filter & $72 \%$ & $12 \%$ & $7 \%$ & $2 \%$ & $1 \%$ \\
\hline Screw Filter & $84 \%$ & $43 \%$ & $10 \%$ & $2 \%$ & $1 \%$ \\
\hline Folded Filter & $52 \%$ & $11 \%$ & $4 \%$ & $1 \%$ & $1 \%$ \\
\hline
\end{tabular}

pletely eliminated. In any case, the filter performance that has been obtained is indeed excellent, and its footprint is greatly reduced.

\section{TOlERANCE ANALYSis}

As it is apparent in all the results presented so far, even though all the filters designed have a wide pass band, they appear to be very sensitive to manufacturing errors. This is an interesting observation since, wide band filters are normally considered to be less sensitive to manufacturing errors. To better understand this issue, we show in this section the results of a yield analysis for all the filters designed and manufactured. To this end, we have used the commercial tool FEST3D to introduce a random error with a Gaussian distribution in the filter dimensions. The computations have been repeated with different values of standard deviation, namely, for $\pm 5, \pm 10, \pm 15, \pm 25$ and $\pm 40 \mu \mathrm{m}$. All filters have been designed with a return loss of $20 \mathrm{~dB}$, and the threshold value for the yield estimation has been set to 18 $\mathrm{dB}$. Table $\mathrm{V}$ shows a summary of the results obtained.

Fig. 37 shows a number of simulations, including errors, for the in-line hybrid SIR filter. It is interesting to note that, from both the simulations and the results in Table V, we can clearly conclude that with manufacturing errors above \pm 25 $\mu \mathrm{m}$ it is impossible to obtain an acceptable in-band response. This result is indeed validated by the prototype manufactured (see Fig. 19), which shows manufacturing errors above 60 microns.

Fig. 38 shows the tolerance analysis of the hybrid SIR filter with body and cover that includes tuning elements in the SIRs. 


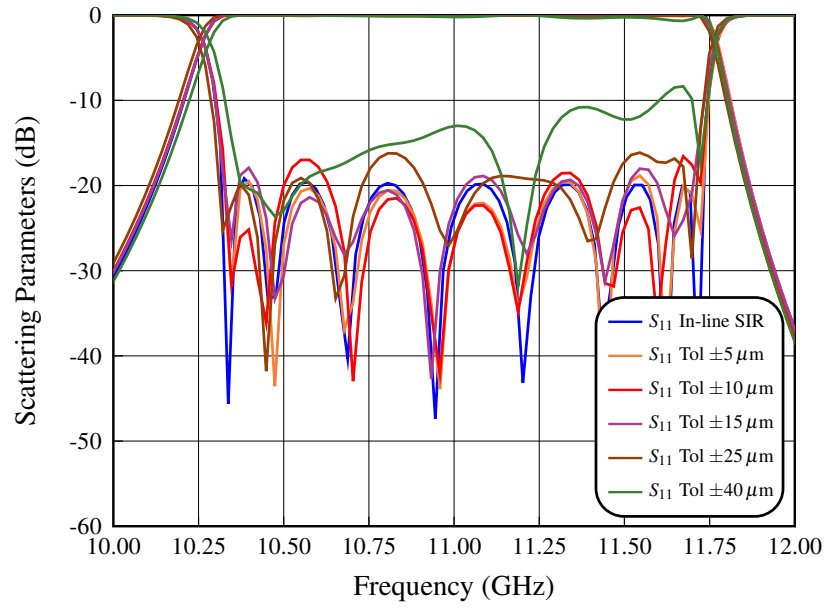

Fig. 37. Tolerance analysis of the in-line hybrid SIR filter.

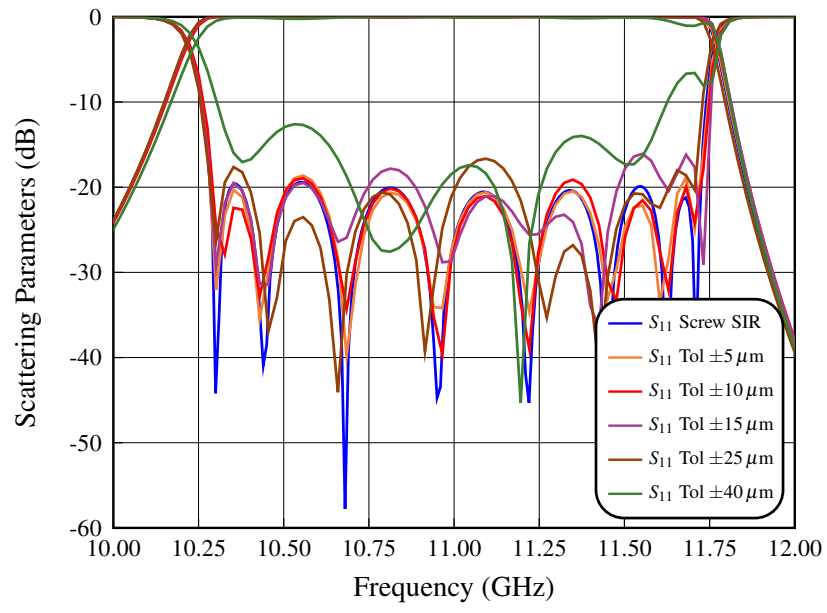

Fig. 38. Tolerance analysis of the in-line hybrid SIR filter with body and cover.

Table V shows that with this structure it should indeed be possible to obtain an acceptable response with manufacturing errors below $\pm 25 \mu \mathrm{m}$, once the tuning elements are properly adjusted. The measured results shown in Fig. 31, however, show a significant degradation of the in-band performance. This is due to the sensitivity of this filter to the dimensions of the coupling irises that could not be tuned. As a result, for manufacturing errors above $\pm 40 \mu \mathrm{m}$ the in-band response can not be recovered even using elements in the resonators. The manufactured filter structure showed again errors in the range of 50 microns, so that an acceptable response could not be obtained even with the use of tuning elements in the resonators. The measured performance is, therefore, in agreement with our yield analysis.

Fig. 39 shows the tolerance analysis of the folded E-plane hybrid SIR filter. From Table V we see that with manufacturing errors less than $10 \mu \mathrm{m}$, the in-band response should show good agreement with respect to the simulations. This is indeed confirmed by the measured results shown in Fig. 35. The measured errors in this filter structure are, in fact, between \pm 5 and $\pm 10 \mu \mathrm{m}$.

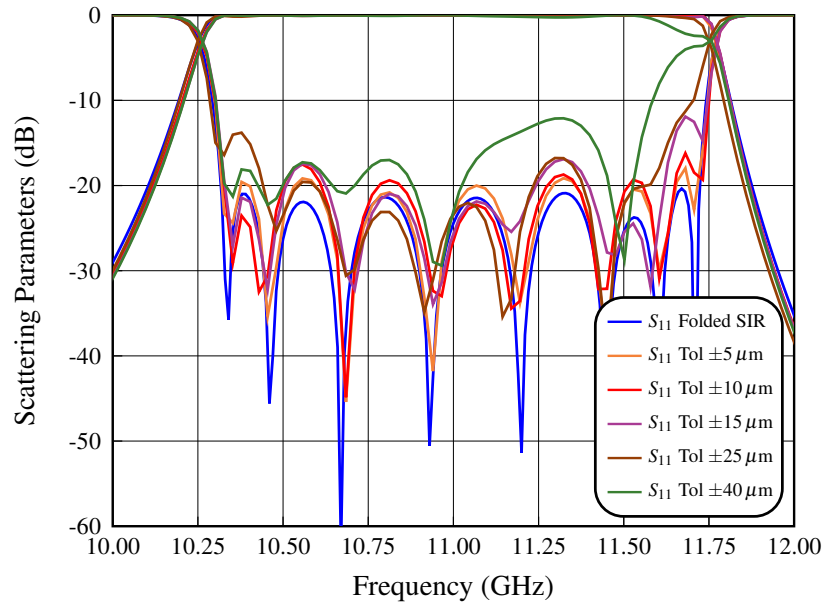

Fig. 39. Tolerance analysis of the folded E-plane hybrid SIR filter.

\section{Conclusions}

In this paper, we have discussed the complete design procedure of a wide band waveguide filter structure with very good out-of-band response. Several designs have been fully described including comparisons between simulation and measurements. A detailed tolerance analysis has also been carried out. Different manufacturing techniques have been explored in order to progressively improve the filter performance. A folded E-plane hybrid SIR filter is finally shown to exhibit the best overall performance. The agreement between simulations and measurements has been shown to be very good in all structures discussed, thereby fully validating both the filter structures and the design processes.

\section{REFERENCES}

[1] R.V. Snyder, "Filter design for modern communication systems," in 2012 Asia Pacific Microwave Conference Proceedings, Dec. 2012, pp. 673675.

[2] R.V. Snyder, A. Mortazawi, I. Hunter, S. Bastioli, G. Macchiarella, and $\mathrm{K}$. Wu, "Present and future trends in filters and multiplexers," IEEE Trans. Microw. Theory Techn., vol. 63, no. 10, pp. 3324-3360, Oct. 2015.

[3] S.B. Cohn, "Analysis of a wide-band waveguide filter," Proceedings of the IRE, vol. 37, no. 6, pp. 651-656, June 1949.

[4] _ , "Design relations for the wide-band waveguide filter," Proceedings of the IRE, vol. 38, no. 7, pp. 799-803, July 1950.

[5] — , "Direct-coupled-resonator filters," Proceedings of the IRE, vol. 45, no. 2, pp. 187-196, Feb. 1957.

[6] G.L. Matthaei, "Design of wide-band (and narrow-band) band-pass microwave filters on the insertion loss basis," IRE Transactions on Microwave Theory and Techniques, vol. 8, no. 6, pp. 580-593, November 1960.

[7] L. Young, "Direct-coupled cavity filters for wide and narrow bandwidths," IEEE Trans. Microw. Theory Techn., vol. 11, no. 3, pp. 162-178, May 1963.

[8] R. Levy, "Theory of direct-coupled-cavity filters," IEEE Trans. Microw. Theory Techn., vol. 15, no. 6, pp. 340-348, June 1967.

[9] — " "A generalized design technique for practical distributed reciprocal ladder networks," IEEE Trans. Microw. Theory Techn., vol. 21, no. 8, pp. 519-526, Aug. 1973.

[10] R. Levy, R.V. Snyder, and G. Matthaei, "Design of microwave filters," IEEE Trans. Microw. Theory Techn., vol. 50, no. 3, pp. 783-793, March 2002.

[11] I.C. Hunter, L. Billonet, B. Jarry, and P. Guillon, "Microwave filtersapplications and technology," IEEE Trans. Microw. Theory Techn., vol. 50, no. 3, pp. 794-805, March 2002. 
[12] R.V. Snyder, "Evolution of passive and active microwave filters," in 2012 IEEE/MTT-S International Microwave Symposium Digest, June 2012, pp. 1-3.

[13] J.F. Liang, K.A. Zaki, and A.E. Atia, "Mixed modes dielectric resonator loaded cavity filters," in 1994 IEEE MTT-S International Microwave Symposium Digest (Cat. No.94CH3389-4), May 1994, pp. 731-734 vol.2.

[14] — " "Mixed modes dielectric resonator filters," IEEE Trans. Microw. Theory Techn., vol. 42, no. 12, pp. 2449-2454, Dec. 1994.

[15] H.W. Yao, K.A. Zaki, A.E. Atia, and R. Hershtig, "Generalized slot coupled combline filters," in Proceedings of 1995 IEEE MTT-S International Microwave Symposium, May 1995, pp. 395-398 vol.2.

[16] J.A. Ruiz-Cruz, K.A. Zaki, J.R. Montejo-Garai, and J.M. Rebollar, "Rectangular waveguide elliptic filters with capacitive and inductive irises and integrated coaxial excitation," in 2005 IEEE/MTT-S International Microwave Symposium Digest, June 2005, pp. 269-272.

[17] A.R. Weily and A.S. Mohan, "Mixed combline and $\mathrm{HE}_{11}$ mode dielectric resonator filter with improved spurious performance," in Proceedings of 1997 Asia-Pacific Microwave Conference, Dec. 1997, pp. 805-808 vol.2.

[18] S. Yuan, X. Wei, G. Yue, and M. Zhang, "Cascaded fourth-order mixed-coupled bandpass filter with good frequency selectivity," in 2013 International Workshop on Microwave and Millimeter Wave Circuits and System Technology, Oct. 2013, pp. 229-232.

[19] P. Chu et al., "A planar bandpass filter implemented with a hybrid structure of substrate integrated waveguide and coplanar waveguide," IEEE Trans. Microw. Theory Techn., vol. 62, no. 2, pp. 266-274, Feb. 2014.

[20] C. Tomassoni, S. Bastioli, and R.V. Snyder, "Mixed-mode resonator using $\mathrm{TE}_{101}$ cavity mode and $\mathrm{TE}_{01 \delta}$ dielectric mode," in 2016 IEEE MTT-S International Microwave Symposium (IMS), May 2016, pp. 1-3.

[21] _ " "Compact mixed-mode filter based on $\mathrm{TE}_{101}$ cavity mode and $\mathrm{TE}_{01 \delta}$ dielectric mode," IEEE Trans. Microw. Theory Techn., vol. 64, no. 12, pp. 4434-4443, Dec. 2016.

[22] K. Li, D. Qu, and X. Zhong, "Novel filters based on hybrid structure of coaxial resonator and evanescent mode waveguide with loaded posts," in 2017 IEEE 9th International Conference on Communication Software and Networks (ICCSN), May 2017, pp. 771-774.

[23] Z. He, Z. Shao, C.J. You, Y.M. Huang, and X. Li, "Design of compact bandpass filter based on quarter-mode substrate integrated waveguide with mixed coupling," in 2015 Asia-Pacific Microwave Conference $(A P M C)$, vol. 3, Dec. 2015, pp. 1-3.

[24] Z. He, C.J. You, S. Leng, X. Li, and Y. Huang, "Compact bandpass filter with high selectivity using quarter-mode substrate integrated waveguide and coplanar waveguide," IEEE Microw. Wireless Compon. Lett., vol. 27, no. 9, pp. 809-811, Sep. 2017.

[25] W.M. Fathelbab, R.V. Snyder, and K.G. Keck, "Wide bandpass filters with wide upper stopbands for high power system applications," in 2011 41st European Microwave Conference, Oct. 2011, pp. 361-364.

[26] S. Bastioli and R.V. Snyder, "Ultra-compact evanescent mode filter using inhomogeneous semi-lumped capacitive elements," in 2014 20th International Conference on Microwaves, Radar and Wireless Communications (MIKON), June 2014, pp. 1-4.

[27] V. Gholipour, S.M.M. Moshiri, A. Alighanbari, and A. Yahaghi, "Highly selective wideband bandpass filter using combined microstrip/coplanar waveguide structure," Electronics Letters, vol. 52, no. 13, pp. 11451147,2016

[28] V. Bagheri, M. Mansouree, K. Mohammadpour-Aghdam, and R. FarajiDana, "Design, construction and measurement of a millimeter-wave filter with 40-60 GHz pass-band," in 2014 Third Conference on MillimeterWave and Terahertz Technologies (MMWATT), Dec. 2014, pp. 1-4.

[29] G.L. Matthaei, L. Young, and E.M.T. Jones, Microwave Filters, Impedance-Matching Networks, and Coupling Structures. Norwood, MA: Artech House, 1980.

[30] S. Li, J. Fu, and X. Wu, "Analysis of high-power rectangular waveguide filter with capacitive coupling iris for satellite," in 2009 Asia-Pacific Power and Energy Engineering Conference, March 2009, pp. 1-4.

[31] _ - "Rectangular waveguide band pass filter with capacitive coupling iris," in Progress in Electromagnetics Research Symposium 2008 (PIERS 2008), March 2008, pp. 337-341.

[32] W. Menzel, A. Muller, and F. Bogelsack, "A capacitively coupled waveguide filter with wide stop-band," in 2003 33rd European Microwave Conference, Oct. 2003, pp. 1239-1242.

[33] M.S. Mahani and M. Tayarani, "Solving complex wave guide structures using hybrid modes and its application in analysis of a wide reject band waveguide iris filter," in 2007 IEEE Applied Electromagnetics Conference (AEMC), Dec. 2007, pp. 1-4.
[34] S. Upadhyay, C. Panchal, P.K. Atrey, and R. Singh, "Development of narrowband microwave bandpass filter for Ku band," in 2016 International Conference on Wireless Communications, Signal Processing and Networking (WiSPNET), March 2016, pp. 893-895.

[35] S. Akatimagool, N. Intarawiset, and S. Inchan, "Design of waveguide bandpass filter using cascade inductive and capacitive irises," in 2016 IEEE 5th Asia-Pacific Conference on Antennas and Propagation (APCAP), July 2016, pp. 387-388.

[36] A. Kirilenko, L. Mospan, and V. Tkachenko, "Capacitive iris bandpass filters with spurious harmonic modes suppression," in International Conference on Mathematical Methods in Electromagnetic Theory, vol. 2, Sep. 2002, pp. 532-534 vol.2.

[37] V.E. Boria et al., "Contributions to the analysis and design of allinductive filters with dielectric resonators," in 33rd European Microwave Conference Proceedings (IEEE Cat. No.03EX723C), vol. 3, Oct. 2003, pp. 1247-1250 Vol.3.

[38] M. Morelli, I. Hunter, R. Parry, and V. Postoyalko, "Stop-band improvement of rectangular waveguide filters using different width resonators: selection of resonator widths," in 2001 IEEE MTT-S International Microwave Sympsoium Digest (Cat. No.01CH37157), vol. 3, May 2001, pp. 1623-1626 vol.3.

[39] N. Yildirim and A. Hizal, "Waveguide filters with ridged and unequal width resonators," in IEEE MTT-S International Microwave Symposium Digest, 2005., June 2005, pp. 1259-1262.

[40] Y. Li, Q. Wang, and C. Wang, "Rectangular waveguide band-pass filter with harmonic suppression," in 2013 International Workshop on Microwave and Millimeter Wave Circuits and System Technology, Oct. 2013, pp. 251-253.

[41] P. Zhao, X. Wang, and C. Chen, "Rectangular waveguide band-pass filter with high power harmonic suppression," in 2014 15th International Conference on Electronic Packaging Technology, Aug. 2014, pp. 13271328.

[42] J.M. Perez Escudero, D. Martinez Martinez, A. Pons Abenza, J.A. Lorente Acosta, A. Alvarez-Melcon, and M. Guglielmi, "Enhancing the spurious free range in inductive rectangular waveguide filters," in 2015 European Microwave Conference (EuMC), Sept. 2015, pp. 678-681.

[43] S. Afridi, M. Sandhu, and I. Hunter, "Mixed non-uniform width/evanescent mode ceramic resonator waveguide filter with wide spurious free bandwidth," in 2016 IEEE MTT-S International Conference on Numerical Electromagnetic and Multiphysics Modeling and Optimization (NEMO), July 2016, pp. 1-3.

[44] S. Afridi, M. Sandhu, N. Somjit, and I. Hunter, "Monolithic ceramic waveguide filter with wide spurious free bandwidth," in 2016 46th European Microwave Conference (EuMC), Oct. 2016, pp. 241-244.

[45] S. Afridi, I. Hunter, and M.Y. Sandhu, "Spurious free non uniform width dielectric loaded filters," in 2018 48th European Microwave Conference (EuMC), Sep. 2018, pp. 85-88.

[46] M. Morelli, I. Hunter, R. Parry, and V. Postoyalko, "Stopband performance improvement of rectangular waveguide filters using steppedimpedance resonators," IEEE Trans. Microw. Theory Techn., vol. 50, no. 7, pp. 1657-1664, July 2002

[47] I. Hunter, S. Afridi, and M. Sandhu, "Integrated ceramic waveguide filters with improved spurious performance," in 2015 European Microwave Conference (EuMC), Sep. 2015, pp. 674-677.

[48] J. Valencia, M. Guglielmi, S. Cogollos, J. Vague, and V.E. Boria, "Enhancing the performance of stepped impedance resonator filters in rectangular waveguide," in 2017 47th European Microwave Conference (EuMC), Oct. 2017, pp. 989-992.

[49] R.J. Cameron, C.M. Kudsia, and R.R. Mansour, Microwave Filters for Communication Systems: Fundamentals, Design and Applications, 2nd ed. Hoboken, NJ, USA: John Wiley \& Sons, 2018.

[50] M. Guglielmi and A.A. Melcon, "Novel design procedure for microwave filters," in 1993 23rd European Microwave Conference, Sept. 1993, pp. $212-213$.

[51] J.W. Bandler, R.M. Biernacki, R.H. Hemmers, and K. Madsen, "Electromagnetic optimization exploiting aggressive space mapping," IEEE Trans. Microw. Theory Techn., vol. 43, no. 12, pp. 2874-2882, Dec. 1995. 


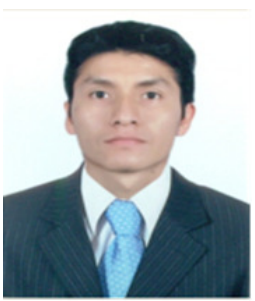

Joaquin Valencia was born in Lima, Peru, on March 14, 1990. He received the degree in telecommunication engineering from the Universidad Nacional de Ingeniería (UNI), Lima, Peru, in 2013, the master degree in communication technologies, systems and networks from the Universitat Politècnica de València, Valencia, Spain, in 2016, where he is currently pursuing the Ph.D. degree in telecommunications with the iTEAM Group. His current research interests include the analysis and design of passive components, electromagnetic simulation, efficient design and optimization of waveguide filters, and its applications.

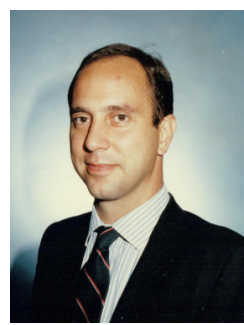

Marco Guglielmi was born in Rome, Italy, on December 17, 1954. He received the degree Laurea in Ingegneria Elettronica in 1979 from the University of Rome La Sapienza, Rome, Italy, where in 1980 he also attended the Scuola di Specializzazione in Elettromagnetismo Applicato. In 1981 he was awarded a Fulbright Scholarship in Rome, Italy, and an HISP (Halsey International Scholarship Programme) from the University of Bridgeport, Bridgeport, Connecticut, USA, where in 1982 he obtained an MS Degree in Electrical Engineering. In 1986 he received a PhD degree in Electrophysics from the Polytechnic University, Brooklyn, New York, USA. From 1984 to 1986 he was Academic Associate at Polytechnic University, and from 1986 to 1988 he was Assistant Professor in the same institution. From 1988 to 1989 he was Assistant Professor at the New Jersey Institute of Technology, Newark, New Jersey, USA. In 1989 he joined the European Space Agency as a Senior Microwave Engineer in the RF System Division of the European Space Research and Technology Centre (ESTEC), Noordwijk, The Netherlands, where he was in charge of the development of microwave filters and electromagnetic simulation tools. In 2001 he was appointed Head of the Technology Strategy Section of ESTEC where he contributed to the development of management processes and tools for the formulation of a European strategy for Space Technology Research and Development. In 2014 Dr. Guglielmi retired from the European Space Agency and is currently holding the position of Invited Senior Researcher at the Polytechnic University of Valencia, Valencia, Spain. Dr. Guglielmi has been elevated to the grade of Fellow of the IEEE in January 2013 For contributions to multimode equivalent networks and microwave filter design.

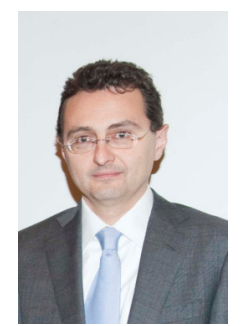

Vicente E. Boria (S'91-A'99-SM'02-F'18) was born in Valencia, Spain, on May 18, 1970. He received his Ingeniero de Telecomunicación degree (with firstclass honors) and the Doctor Ingeniero de Telecomunicación degree from the Universidad Politécnica de Valencia, Valencia, Spain, in 1993 and 1997, respectively. In 1993 he joined the Departamento de Comunicaciones, Universidad Politécnica de Valencia, where he has been Full Professor since 2003. In 1995 and 1996, he was holding a Spanish Trainee position with the European Space Research and Technology Centre, European Space Agency (ESTEC-ESA), Noordwijk, The Netherlands, where he was involved in the area of EM analysis and design of passive waveguide devices. He has authored or co-authored 10 chapters in technical textbooks, 180 papers in refereed international technical journals, and over 200 papers in international conference proceedings. His current research interests are focused on the analysis and automated design of passive components, left-handed and periodic structures, as well as on the simulation and measurement of power effects in passive waveguide systems. Dr. Boria has been a member of the IEEE Microwave Theory and Techniques Society (IEEE MTT-S) and the IEEE Antennas and Propagation Society (IEEE AP-S) since 1992. He is also member of the European Microwave Association (EuMA), and has been the Chair of the 48th European Microwave Conference held in Madrid, Spain. He acts as a regular reviewer of the most relevant IEEE and IET technical journals on his areas of interest. He has been Associate Editor of IEEE Microwave and Wireless Components Letters (2013-2018) and IET Electronics Letters (2015-2018). Presently, he serves as Subject Editor (Microwaves) of IET Electronics Letters, and as Editorial Board member of International Journal of RF and Microwave Computer-Aided Engineering. He is also member of the Technical Committees of the IEEE-MTT International Microwave Symposium and of the European Microwave Conference.

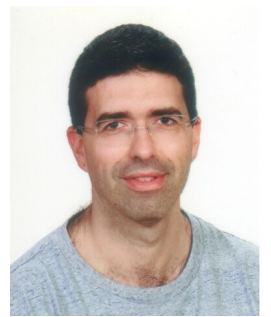

Santiago Cogollos was born in Valencia, Spain, on January 15,1972 . He received the degree in telecommunication engineering and the $\mathrm{Ph}$. D. degree from the Universitat Politècnica de València (UPV), Valencia, Spain, in 1996 and 2002, respectively. In 2000 he joined the Communications Department of the Universitat Politècnica de València, where he was an Assistant Lecturer from 2000 to 2001 , a Lecturer from 2001 to 2002, and became an Associate Professor in 2002. He has collaborated with the European Space Research and Technology Centre of the European Space Agency in the development of modal analysis tools for payload systems in satellites. In 2005 he held a post doctoral research position working in the area of new synthesis techniques in filter design at University of Waterloo, Waterloo, Ont., Canada. His current research interests include applied electromagnetics, mathematical methods for electromagnetic theory, analytical and numerical methods for the analysis of microwave structures, and design of waveguide components for space applications. 